\title{
A 'User-Friendly' Approach to the Dynamical Equations of Non-Holonomic Systems ${ }^{\star}$
}

Sergio BENENTI

Department of Mathematics, University of Turin, Italy

E-mail: sergio.benenti@unito.it

URL: http://www2.dm.unito.it/ benenti/

Received November 29, 2006, in final form February 13, 2007; Published online March 01, 2007

Original article is available at http://www.emis.de/journals/SIGMA/2007/036/

\begin{abstract}
Two effective methods for writing the dynamical equations for non-holonomic systems are illustrated. They are based on the two types of representation of the constraints: by parametric equations or by implicit equations. They can be applied to linear as well as to non-linear constraints. Only the basic notions of vector calculus on Euclidean 3-space and on tangent bundles are needed. Elementary examples are illustrated.
\end{abstract}

Key words: non-holonomic systems; dynamical systems

2000 Mathematics Subject Classification: 37J60; 70F25

\section{Preamble}

The classical theory of non-holonomic dynamical systems, even in recent times, is treated in a growing number of papers. Most of them require the use of modern differentiable and algebraic structures which are not familiar to non-mathematicians working on concrete applications. On the other hand, several papers are dedicated to the analysis of special non-holonomic mechanical systems, quite interesting but treated with ad hoc methods. These are the reasons why I think useful to propose a ready to use approach to the dynamics of non-holonomic systems, requiring the knowledge of the basic notions of the vector calculus on the Euclidean three-space and on tangent bundles only, and avoiding the use of cotangent bundles (Hamiltonian framework) and jet-bundles.

In the present paper I illustrate two different, general, effective and concise methods for writing the dynamical equations of a given non-holonomic system. These methods correspond to the two ways of representing kinematical constraints: by parametric equations or by implicit equations. They can be applied to linear as well as to non-linear constraints and lead to different (but obviously equivalent) dynamical systems.

In order to make this paper self-contained, a straightforward approach to the Gauss principle and to the Gibbs-Appell equations is illustrated. Our starting point will be the well understandable Newton dynamical equations for a system of massive points.

The tutorial character of this paper does not exclude the presence of some novelties.

Many articles and books have been consulted in writing this paper. To cite all them would make the list of references quite long. On the other hand, such a long list would not fit with the purposes of this paper. Anyway, I must mention the excellent recent books [2, 4], from which I have got many useful hints. They are very well readable and advisable to non-experts which would like to go into this matter in more depth, and to know its present developments and applications.

\footnotetext{
*This paper is a contribution to the Vadim Kuznetsov Memorial Issue "Integrable Systems and Related Topics". The full collection is available at http://www.emis.de/journals/SIGMA/kuznetsov.html
} 


\section{Introduction}

Let us consider a mechanical system with a well defined configuration manifold $Q_{n}$. The dimension $n$ of $Q$ is the number of degrees of freedom of the system. Let us denote by $q=\left(q^{i}\right)$ any (Lagrangian) coordinate system on $Q$ and by $(q, \dot{q})=\left(q^{i}, \dot{q}^{i}\right)$ the associated coordinates on the tangent bundle $T Q$. This tangent bundle is the space of the kinematical states.

A kinematical constraint is given by a subset $C \subset T Q$. A special case is that of regular constraint:

Definition 2.1. A kinematical constraint $C \subset T Q$ is said to be regular if

- $C$ is a submanifold of dimension $n+m, m<n$;

- for all $q \in Q, F_{q}=C \cap T_{q} Q$ is a submanifold of dimension $m$;

- the restriction to $C$ of the tangent fibration $\tau_{Q}: T Q \rightarrow Q$ is a surjective submersion ${ }^{1}$.

The constraint is linear if each $F_{q}$ is a subspace (see Fig. 1).

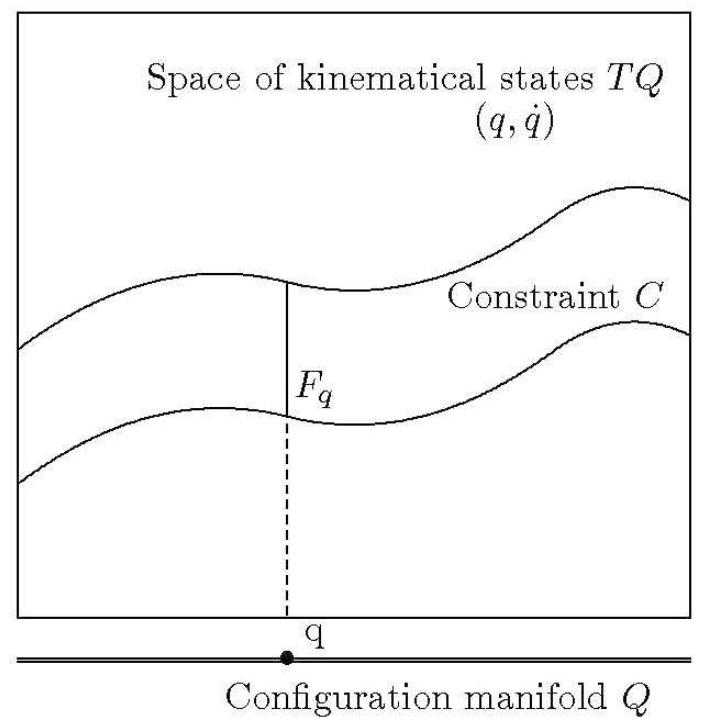

Figure 1. A regular constraint is a fibration onto $Q$.

A regular constraint can be represented in two ways:

- Parametric representation: it is described by $m$ equations,

$$
\dot{q}^{i}=\psi^{i}(q, z),
$$

where $z=\left(z^{\alpha}\right), \alpha=1, \ldots, m<n$ are called parameters. Note that $(q, z)$ can be interpreted as local coordinates on $C$.

- Implicit representation: it is described by $r=n-m$ independent equations ${ }^{2}$,

$$
C^{a}(q, \dot{q})=0, \quad a=1, \ldots, r .
$$

\footnotetext{
${ }^{1}$ If this notion is not understood by the reader, he can look at the equivalent conditions (2.3) and (2.4) below. The definition of regularity is taken from [10] and [6]. In [6] an extension of this definition is given: it requires the existence of a submanifold $Q_{1} \subset Q$ such that $C$ is a submanifold of $T Q_{1}$ and the restriction to $C$ of the tangent fibration is a submersion. For our purposes we do not need to consider this more general case.

${ }^{2}$ This means that the differentials $d C^{a}$ are linearly independent at each point of $C$.
} 
In these two representations, the regularity of the constraint is represented by conditions

$$
\operatorname{rank}\left[\psi_{\alpha}^{i}\right]_{n \times m}=m, \quad \operatorname{rank}\left[C_{i}^{a}\right]_{n \times r}=r,
$$

respectively, where

$$
\psi_{\alpha}^{i} \doteq \frac{\partial \psi^{i}}{\partial z^{\alpha}}, \quad C_{i}^{a} \doteq \frac{\partial C^{a}}{\partial \dot{q}^{i}}
$$

Note that the regularity conditions may be not fulfilled at certain states, that we call singular states. They always occurs, for instance, for non-linear homogeneous constraints (see Remark 7.5).

In the following, with the exception of special remarks, the constraints are assumed to be nonlinear. However, in the case of linear constraints, we shall assume - without loss of generality that the functions $\psi^{i}(q, z)$ are linear in the parameters, so that $\psi^{i}=\psi_{\alpha}^{i}(q) z^{\alpha}$, or that the functions $C^{a}(q, \dot{q})$ are linear in the $\dot{q}$, so that $C^{a}=C_{i}^{a}(q) \dot{q}^{i}$.

The leading idea of the first method is to consider the parametric equations (2.1) of the constraint as a first set of dynamical equations, to be completed by a second set of equations of the kind $\dot{z}^{\alpha}=Z^{\alpha}(q, z)$ :

$$
\begin{aligned}
& \dot{q}^{i}=\psi^{i}(q, z), \\
& \dot{z}^{\alpha}=Z^{\alpha}(q, z) .
\end{aligned}
$$

This is a first-order system of ODE's. Once the initial conditions $\left(q_{0}, z_{0}\right)$ are fixed, they give a unique actual motion of the mechanical system. By actual motion we mean a physical motion obeying to the constraints.

The explicit expressions of the functions $Z^{\alpha}(q, z)$ depend of course on the given functions $\psi^{i}(q, z)$. But they depend also on the dynamical principles we assume.

The Newton dynamical equation for a material point will be the only physical principle on which we shall base our approach. Furthermore, we shall assume that the constraints are ideal or perfect, according to a suitable mathematical definition: this means to accept a certain constitutive condition on the constraint as a postulate. In this way, the Gauss 'principle' will follow as a 'theorem' from the Newton equations of a system of particles.

The second method presented here is based on the implicit representation of the constraints. It is a revisitation, with improvements and simplifications, of the well-known Lagrangemultipliers method. In this context we get the explicit expression of the reactive forces. This is useful, in the concrete applications, for measuring the stress that the constraints have to support.

These two methods lead to different first-order dynamical equations i.e., to different vector fields:

- The first method (parametric representation) produces a vector field $\mathbf{Z}$ on the constraint manifold $C$. The integral curves of $\mathbf{Z}$ give all actual motions - see Fig. 2.

- The second method (implicit representation) produces a vector field $\mathbf{D}$ on the whole $T Q$ but tangent to $C$. Only its restriction to $C$ has in fact a physical meaning i.e., only its integral curves which start from a point of $C$ (and which will lie on $C$ ) represent actual motions - see Fig. 3. As a byproduct, this method gives the explicit expressions of the reactive forces, which can be estimated along any actual motion. 


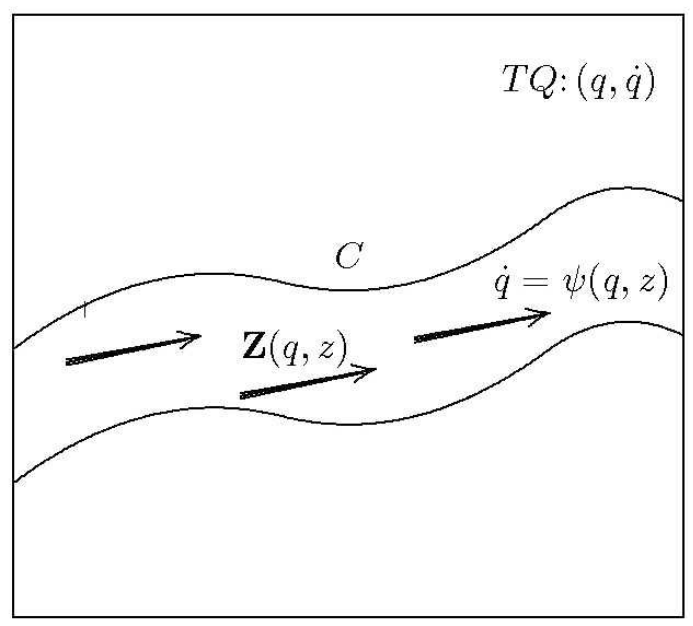

$Q:(q)$

Figure 2. Parametric representation of $C$ - The vector field $\mathbf{Z}$.

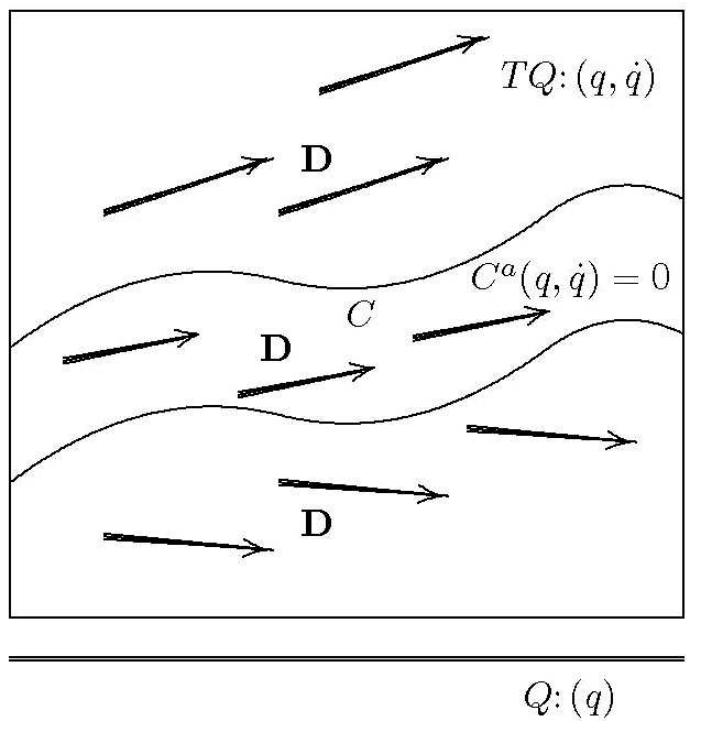

Figure 3. Implicit representation of $C$ - The vector field $\mathbf{D}$.

\section{Ideal constraints}

At a microscopical level, a mechanical system is made of a collection of material points $\left(P_{\nu}, m_{\nu}\right)$, $\nu=1, \ldots, N$. The position vector $\mathbf{r}_{\nu}$ of each point $P_{\nu}$ in the Euclidean three-space is a function $\mathbf{r}_{\nu}(q)$ of the chosen Lagrangian coordinates. A motion of the system is then represented by a time-parametrized curve $q^{i}=q^{i}(t)$ on $Q$, and at each configuration $q \in Q$ all possible velocities of the points are given by

$$
\mathbf{v}_{\nu}=\frac{\partial \mathbf{r}_{\nu}}{\partial q^{i}} \dot{q}^{i}, \quad\left(v^{i}\right) \in \mathbb{R} .
$$

A kinematical state of the system is the collection of all pairs position-velocity $\left(\mathbf{r}_{\nu}, \mathbf{v}_{\nu}\right)$ of the points. The collection of all possible states is then the tangent bundle $T Q$ of $Q$. At any fixed state the accelerations $\mathbf{a}_{\nu}$ are given by

$$
\mathbf{a}_{\nu}=\frac{\partial^{2} \mathbf{r}_{\nu}}{\partial q^{i} \partial q^{j}} \dot{q}^{i} \dot{q}^{j}+\frac{\partial \mathbf{r}_{\nu}}{\partial q^{i}} \frac{d \dot{q}^{i}}{d t} .
$$

Let us consider the parametric representation (2.1) of the constraint. Then the velocity and the acceleration of each point, compatible with the kinematical constraint, are

$$
\begin{aligned}
& \mathbf{v}_{\nu}(q, z)=\frac{\partial \mathbf{r}_{\nu}}{\partial q^{i}} \psi^{i} \\
& \mathbf{a}_{\nu}(q, z, \dot{z})=\frac{\partial^{2} \mathbf{r}_{\nu}}{\partial q^{i} \partial q^{j}} \psi^{i} \psi^{j}+\frac{\partial \mathbf{r}_{\nu}}{\partial q^{i}}\left(\frac{\partial \psi^{i}}{\partial q^{j}} \psi^{j}+\frac{\partial \psi^{i}}{\partial z^{\alpha}} \dot{z}^{\alpha}\right) .
\end{aligned}
$$

Let us write these equations in the more compact form, according to the above-given notation:

$$
\begin{aligned}
& \mathbf{v}_{\nu}(q, z)=\partial_{i} \mathbf{r}_{\nu} \psi^{i} \\
& \mathbf{a}_{\nu}(q, z, \dot{z})=\partial_{i j} \mathbf{r}_{\nu} \psi^{i} \psi^{j}+\partial_{i} \mathbf{r}_{\nu}\left(\psi_{j}^{i} \psi^{j}+\psi_{\alpha}^{i} \dot{z}^{\alpha}\right) .
\end{aligned}
$$

As we shall see, the additional parameters $\dot{z}$ play a crucial role. Note that, at any fixed configuration $q \in Q$, the parameters $z$ determine all kinematical states $(q, v)$ compatible with 
the constraints. Intuitively, the parameters $\dot{z}$ determine a small displacement from a state $(q, \dot{q})$ to another close state $\left(q, \dot{q}^{\prime}\right)$ (with the same configuration $q$ ). For a precise definition, let us decompose the acceleration (3.3) into the sum

$$
\mathbf{a}_{\nu}=\mathbf{a}_{0 \nu}+\mathbf{a}_{\alpha \nu} \dot{z}^{\alpha}
$$

where

$$
\mathbf{a}_{0 \nu} \doteq \partial_{i j} \mathbf{r}_{\nu} \psi^{i} \psi^{j}+\partial_{i} \mathbf{r}_{\nu} \psi_{j}^{i} \psi^{j}, \quad \mathbf{a}_{\alpha \nu} \doteq \partial_{i} \mathbf{r}_{\nu} \psi_{\alpha}^{i}
$$

We observe that the vectors $\mathbf{a}_{0 \nu}$ depend only on the state of the system. We say that

Definition 3.1. The second vector in (3.4)

$$
\mathbf{w}_{\nu} \doteq \mathbf{a}_{\alpha \nu} \dot{z}^{\alpha}
$$

represents the virtual displacements of the point $P_{\nu}$ at the given kinematical state.

Remark 3.1. Note that the parameters $\dot{z}^{\alpha}$ span all possible virtual displacements at a given state. The mechanical meaning of virtual displacement, which is strictly related to that of ideal constraint (Definition 3.2) would require a detailed discussion. We can skip it simply by accepting equation (3.6) as a mathematical definition, since it will be justified first, by the fact that with such a definition the Gauss principle becomes a consequence of the Newton dynamical equations (Theorem 4.1) and second, by the fact that the reactive forces of ideal constraints are not dissipative (Remark 3.3).

It is customary to associate the intuitive idea of 'virtual displacement' with that of 'virtual velocity', as a limit of a 'small' displacement between two configurations' of the system. Instead, within the present context, a 'virtual displacement' is a 'small' displacement between kinematical states (configurations plus velocities), so it is associated with the intuitive idea of "virtual acceleration'. This viewpoint is in fact coherent with the philosophy of the Gauss principle, which deals with accelerations.

We assume for the dynamics of each point $\left(P_{\nu}, m_{\nu}\right)$ the Newton equation

$$
m_{\nu} \mathbf{a}_{\nu}=\mathbf{A}_{\nu}+\mathbf{R}_{\nu}
$$

where $\mathbf{A}_{\nu}$ is the active force (due to external fields and internal interactions) and $\mathbf{R}_{\nu}$ is the reactive force: it has the role of making the constraint fulfilled.

Remark 3.2. The idea of 'reactive force' arises from the Newtonian philosophy, according which any action deviating a point from the uniform rectilinear motion (in an inertial reference frame) is a 'force', mathematically represented by a vector. Thus, the presence of a kinematical constraint must be represented by a vector, called 'reactive force', to be summed to the 'active force', which in turns represents the action of fields present in the space and independent from the constraints (gravitational, electromagnetical, centrifugal, Coriolis, etc.).

Definition 3.2. Non-holonomic constraints are said to be ideal or perfect if

$$
\sum_{\nu} \mathbf{R}_{\nu} \cdot \mathbf{w}_{\nu}=0
$$

for all virtual displacements $\mathbf{w}_{\nu}$. 
We consider equation (3.8) as a constitutive condition for the constraint: it says which kind of reactive forces the constraint is able to supply in order to be satisfied along any motion. It is straightforward to check that for linear constraints, or simply for holonomic constraints (which do not involve velocities), equation (3.8) reduces to the classical virtual work principle. The validity of such a constitutive condition is a matter of theoretical and experimental analysis of the behavior of the mechanical system one is dealing with.

Theorem 3.1. Let

$$
R_{i} \doteq \sum_{\nu} \mathbf{R}_{\nu} \cdot \frac{\partial \mathbf{r}_{\nu}}{\partial q^{i}}
$$

be the Lagrangian reactive forces. Then the definition (3.8) of ideal constraint is equivalent to the following equations,

$$
\begin{aligned}
& R_{i} \psi_{\alpha}^{i}=0, \\
& R_{i}=\lambda_{a} C_{i}^{a},
\end{aligned}
$$

in the parametric and in the implicit representation, respectively.

Equation (3.11) means that the components $R_{i}$ are linear combinations of the functions $C_{i}^{a}(q, \dot{q})$.

Proof. Put equation (3.6) and (3.5) in equation (3.8),

$$
0=\sum_{\nu} \mathbf{R}_{\nu} \cdot \mathbf{a}_{\alpha \nu} \dot{z}^{\alpha}=\sum_{\nu} \mathbf{R}_{\nu} \cdot \partial_{i} \mathbf{r}_{\nu} \psi_{\alpha}^{i} \dot{z}^{\alpha}=R_{i} \psi_{\alpha}^{i} \dot{z}^{\alpha} .
$$

This proves equation (3.10). By differentiating the identity $C^{a}(q, \psi(q, z))=0$, we get the following relations between the two representations,

$$
C_{i}^{a} \psi_{\alpha}^{i}=0, \quad \partial_{i} C^{a}+C_{j}^{a} \psi_{i}^{j}=0,
$$

where

$$
C_{i}^{a} \doteq \frac{\partial C^{a}}{\partial \dot{q}^{i}}, \quad \psi_{i}^{j} \doteq \partial_{i} \psi^{j}, \quad \partial_{i} \doteq \frac{\partial}{\partial q^{i}} .
$$

Due to the first identity (3.12), equation (3.10) is then equivalent to (3.11)

Remark 3.3. Equations $(3.13)_{1}$ and (3.11) show that the ideal constraints do not dissipate energy: the power of the reactive forces is zero (we are dealing with time-independent constraints). See [3].

Remark 3.4. By a well-known process, we pass from the microscopical level to the ' $m a c r o s c o$ pical' one i.e., to the Lagrange equations

$$
\frac{d}{d t}\left(\frac{\partial K}{\partial \dot{q}^{i}}\right)-\frac{\partial K}{\partial q^{i}}=A_{i}+R_{i}
$$

where

$$
K=\frac{1}{2} g_{i j} \dot{q}^{i} \dot{q}^{j}, \quad g_{i j} \doteq \sum_{\nu} m_{\nu} \partial_{i} \mathbf{r}_{\nu} \cdot \partial_{j} \mathbf{r}_{\nu}
$$

At the right hand side of the Lagrange equations we find the sum of the active Lagrangian forces

$$
A_{i} \doteq \sum_{\nu} \mathbf{A}_{\nu} \cdot \frac{\partial \mathbf{r}_{\nu}}{\partial q^{i}}
$$

and the reactive Lagrangian forces (3.9). 


\section{The Gauss principle}

At the microscopical level we introduce the quantity

$$
G \doteq \frac{1}{2} \sum_{\nu} m_{\nu}\left(\mathbf{a}_{\nu}-\frac{\mathbf{A}_{\nu}}{m_{\nu}}\right)^{2}
$$

The active forces $\mathbf{A}_{\nu}$ are known functions of the state $(q, \dot{q})$. Thus, due to the parametric equations of the constraints, $G$ becomes a function of $(q, z)$. Moreover, even for active forces depending on the velocities, $\mathbf{A}_{\nu}$ does not depend on $\dot{z}$,

$$
\frac{\partial \mathbf{A}_{\nu}}{\partial \dot{z}^{\alpha}}=0
$$

Thus, due to equations (3.4) and (3.5), along any motion satisfying the constraints we have

$$
\frac{\partial G}{\partial \dot{z}^{\alpha}}=\sum_{\nu} m_{\nu}\left(\mathbf{a}_{\nu}-\frac{\mathbf{A}_{\nu}}{m_{\nu}}\right) \cdot \frac{\partial \mathbf{a}_{\nu}}{\partial \dot{z}^{\alpha}}=\sum_{\nu} m_{\nu}\left(\mathbf{a}_{\nu}-\frac{\mathbf{A}_{\nu}}{m_{\nu}}\right) \cdot \mathbf{a}_{\alpha \nu} .
$$

Furthermore,

$$
\frac{\partial^{2} G}{\partial \dot{z}^{\alpha} \partial \dot{z}^{\beta}}=\sum_{\nu} m_{\nu} \frac{\partial \mathbf{a}_{\nu}}{\partial \dot{z}^{\beta}} \cdot \mathbf{a}_{\alpha \nu}=\sum_{\nu} m_{\nu} \mathbf{a}_{\beta \nu} \cdot \mathbf{a}_{\alpha \nu}=\sum_{\nu} m_{\nu} \psi_{\alpha}^{i} \psi_{\beta}^{j} \partial_{i} \mathbf{r}_{\nu} \cdot \partial_{j} \mathbf{r}_{\nu}=g_{i j} \psi_{\alpha}^{i} \psi_{\beta}^{j} .
$$

Then, if we introduce the functions

$$
G_{\alpha \beta} \doteq g_{i j} \psi_{\alpha}^{i} \psi_{\beta}^{j},
$$

we get

$$
\frac{\partial^{2} G}{\partial \dot{z}^{\alpha} \partial \dot{z}^{\beta}}=G_{\alpha \beta}
$$

Since the matrix $\left[\psi_{\alpha}^{i}\right]$ has maximal rank, the symmetric matrix $\left[G_{\alpha \beta}\right]$ is regular and positivedefinite as well as $\left[g_{i j}\right]$.

Theorem 4.1. Assume the Newton equations $m_{\nu} \mathbf{a}_{\nu}=\mathbf{A}_{\nu}+\mathbf{R}_{\nu}$ for each point $P_{\nu}$. Then, at any state of any actual motion the quantity $G$ takes a minimal value (Gauss principle) if and only if the constraints are ideal.

Proof. Write the Newton equations in the form

$$
m_{\nu}\left(\mathbf{a}_{\nu}-\frac{\mathbf{A}_{\nu}}{m_{\nu}}\right)=\mathbf{R}_{\nu}
$$

Then, due to equations (3.4), (3.5) and (3.9),

$$
\frac{\partial G}{\partial \dot{z}^{\alpha}}=\sum_{\nu} \mathbf{R}_{\nu} \cdot \frac{\partial \mathbf{a}_{\nu}}{\partial \dot{z}^{\alpha}}=\sum_{\nu} \mathbf{R}_{\nu} \cdot \mathbf{a}_{\alpha \nu}=R_{i} \psi_{\alpha}^{i} .
$$

This shows that for ideal constraints, see equation (3.10), the Newton equations imply

$$
\frac{\partial G}{\partial \dot{z}^{\alpha}}=0
$$

at any state along any actual motion. Due to equation (4.4), being $\left[G_{\alpha \beta}\right]$ positive, at the stationary states for which equation (4.6) holds, the function $G$ has a strong minimum. (ii) Conversely, assume that the Gauss principle holds true. Then equation (4.6) is satisfied, so that from (4.5) we get $R_{i} \psi_{\alpha}^{i}=0$. This means that the constraint is ideal (Theorem 3.1). 
Remark 4.1. The vector $\mathbf{A}_{\nu} / m_{\nu}$ is the acceleration of the point $P_{\nu}$ in a free motion, free from the constraints. Let us denote it by $\mathbf{a}_{\nu}^{f}$. As a consequence, the function $G$ can be also defined as

$$
G \doteq \frac{1}{2} \sum_{\nu} m_{\nu}\left(\mathbf{a}_{\nu}-\mathbf{a}_{\nu}^{f}\right)^{2}
$$

and Theorem 4.1 can be reformulated as follows:

Theorem 4.2. Let $\mathbf{r}_{\nu}(t)$ and $\mathbf{r}_{\nu}^{f}(t)$ be two motions of the system $P_{\nu}$ such that for $t=t_{0}$ the corresponding states coincide i.e.,

$$
\mathbf{r}_{\nu}\left(t_{0}\right)=\mathbf{r}_{\nu}^{f}\left(t_{0}\right), \quad \mathbf{v}_{\nu}\left(t_{0}\right)=\mathbf{v}_{\nu}^{f}\left(t_{0}\right) .
$$

Assume that $\mathbf{r}_{\nu}^{f}(t)$ is a free motion. Then, at this state, and for any motion compatible with ideal constraints, the actual accelerations $\mathbf{a}_{\nu}\left(t_{0}\right)$, are such that $G$ takes a minimal value.

Remark 4.2. For any arbitrary motion,

$$
\mathbf{v}_{\nu}=\frac{\partial \mathbf{r}_{\nu}}{\partial q^{i}} \dot{q}^{i}, \quad \mathbf{a}_{\nu}=\frac{\partial \mathbf{r}_{\nu}}{\partial q^{i} \partial q^{j}} \dot{q}^{i} \dot{q}^{j}+\frac{\partial \mathbf{r}_{\nu}}{\partial q^{i}} \ddot{q}^{i} .
$$

Then, at any fixed state compatible with the constraints we have

$$
\mathbf{a}_{\nu}-\mathbf{a}_{\nu}^{f}=\frac{\partial \mathbf{r}_{\nu}}{\partial q^{i}}\left(\ddot{q}^{i}-\ddot{q}_{f}^{i}\right) .
$$

Due to the definition (3.15) of $g_{i j}$, from (4.7) we get the so-called Lipschitz expression of the function $G$ :

$$
G=\frac{1}{2} g_{i j}\left(\ddot{q}^{i}-\ddot{q}_{f}^{i}\right)\left(\ddot{q}^{j}-\ddot{q}_{f}^{j}\right) .
$$

Note that in this expression the Christoffel symbols are not involved.

\section{The Gibbs-Appell equations}

Let us go back to the definition (4.1) of the function $G$. If we introduce the functions

$$
S \doteq \frac{1}{2} \sum_{\nu} m_{\nu} \mathbf{a}_{\nu}^{2}, \quad S_{1} \doteq \frac{1}{2} \sum_{\nu} \frac{1}{m_{\nu}} \mathbf{A}_{\nu}^{2}, \quad S_{2} \doteq \sum_{\nu} \mathbf{A}_{\nu} \cdot \mathbf{a}_{\nu}
$$

then we have the decomposition

$$
G=S+S_{1}-S_{2} .
$$

The function $S$ is called the energy of the accelerations. We observe that

$$
\frac{\partial S_{1}}{\partial \dot{z}^{\alpha}}=0
$$

and that, due to the second equation (3.3) and the definition (3.16) of active Lagrangian force,

$$
\frac{\partial S_{2}}{\partial \dot{z}^{\alpha}}=\sum_{\nu} \mathbf{A}_{\nu} \cdot \partial_{i} \mathbf{r}_{\nu} \psi_{\alpha}^{i}=A_{i} \psi_{\alpha}^{i}
$$

Thus,

$$
\frac{\partial G}{\partial \dot{z}^{\alpha}}=\frac{\partial S}{\partial \dot{z}^{\alpha}}-A_{i} \psi_{\alpha}^{i}
$$

Due to the Gauss principle (Theorem 4.1), this proves 
Theorem 5.1. The Gauss principle is equivalent to equations

$$
\frac{\partial S}{\partial \dot{z}^{\alpha}}=A_{\alpha}
$$

where the function

$$
S(q, z, \dot{z}) \doteq \frac{1}{2} \sum_{\nu} m_{\nu} \mathbf{a}_{\nu}^{2}
$$

is determined by the expression (3.3) of the accelerations, and

$$
A_{\alpha} \doteq A_{i} \psi_{\alpha}^{i}
$$

Remark 5.1. Equations (5.2) are the celebrated Gibbs-Appell equations. The equivalence between these equations and the Gauss principle is highlighted within the framework of the parametric representation (2.1) of the constraints.

Remark 5.2. The quantities $A_{\alpha}$ can be computed by writing the the virtual power of the active forces:

$$
W \doteq \sum_{\nu} \mathbf{A}_{\nu} \cdot \mathbf{w}_{\nu}=\sum_{\nu} \mathbf{A}_{\nu} \cdot \partial_{i} \mathbf{r}_{\nu} \psi_{\alpha}^{i} \dot{z}^{\alpha}=A_{i} \psi_{\alpha}^{i} \dot{z}^{\alpha}=A_{\alpha} \dot{z}^{\alpha}
$$

\section{The explicit form of the Gibbs-Appell equations}

Both sides of the Gibbs-Appell equations (5.2) are functions of $(q, z, \dot{z})$. Let us solve them w.r.to the variables $\dot{z}^{\alpha}$. To this end, it is crucial to observe that by using the second equations (3.3) we get for the function $S$ (5.3) the expression

$$
S=\frac{1}{2} g_{i j} \psi_{\alpha}^{i} \psi_{\beta}^{j} \dot{z}^{\alpha} \dot{z}^{\beta}+\sum_{\nu} m_{\nu} \partial_{i j} \mathbf{r}_{\nu} \cdot \partial_{k} \mathbf{r}_{\nu} \psi^{i} \psi^{j} \psi_{\alpha}^{k} \dot{z}^{\alpha}+S_{0},
$$

where $S_{0}$ is a function dependent on $(q, z)$ only. Then, this function is not involved by the Gibbs-Appell equations and $S$ can be replaced by

$$
S_{*}=\frac{1}{2} g_{i j} \psi_{\alpha}^{i} \psi_{\beta}^{j} \dot{z}^{\alpha} \dot{z}^{\beta}+\sum_{\nu} m_{\nu} \partial_{i j} \mathbf{r}_{\nu} \cdot \partial_{k} \mathbf{r}_{\nu} \psi^{i} \psi^{j} \psi_{\alpha}^{k} \dot{z}^{\alpha} .
$$

Now we show that this new function $S_{*}$ assumes a very interesting expression. Let us introduce the functions

$$
\xi_{i j k}(q) \doteq \sum_{\nu} m_{\nu} \partial_{i j} \mathbf{r}_{\nu} \cdot \partial_{k} \mathbf{r}_{\nu}
$$

Since

$$
\xi_{i j k}=\sum_{\nu} m_{\nu} \partial_{i}\left(\partial_{j} \mathbf{r}_{\nu} \cdot \partial_{k} \mathbf{r}_{\nu}\right)-\sum_{\nu} m_{\nu}\left(\partial_{j} \mathbf{r}_{\nu} \cdot \partial_{i k} \mathbf{r}_{\nu}\right)=\partial_{i} g_{j k}-\xi_{i k j},
$$

by a cyclic permutation of the indices we get

$$
\xi_{i j k}+\xi_{i k j}=\partial_{i} g_{j k}, \quad \xi_{j k i}+\xi_{j i k}=\partial_{j} g_{k i}, \quad \xi_{k i j}+\xi_{k j i}=\partial_{k} g_{i j} .
$$

By summing the first two equations and subtracting the third one, since $\xi_{i j k}$ is symmetric in the first two indices, we get $\xi_{i j k}=\Gamma_{i j k}$, where

$$
\Gamma_{i j k} \doteq \frac{1}{2}\left(\partial_{i} g_{j k}+\partial_{j} g_{k i}-\partial_{k} g_{i j}\right)
$$


are the Christoffel symbols of the metric tensor $g_{i j}$ (the coefficients of the Levi-Civita connection). As a consequence, if we recall the definition (4.3) of $G_{\alpha \beta}$, the function $S_{*}$ (6.1) can be written as

$$
S_{*}=\frac{1}{2} G_{\alpha \beta} \dot{z}^{\alpha} \dot{z}^{\beta}+\Gamma_{i j k} \psi^{i} \psi^{j} \psi_{\alpha}^{k} \dot{z}^{\alpha},
$$

and the Gibbs-Appell equations (5.2) assume the form

$$
G_{\alpha \beta} \dot{z}^{\beta}+\Gamma_{i j k} \psi^{i} \psi^{j} \psi_{\alpha}^{k}=A_{\alpha} .
$$

Then we can prove

Theorem 6.1. The Gibbs-Appell equations (5.2) are equivalent to equations

$$
\dot{z}^{\alpha}=G^{\alpha \beta}\left(A_{\beta}-\Gamma_{i j k} \psi^{i} \psi^{j} \psi_{\beta}^{k}\right),
$$

where $\left[G^{\alpha \beta}\right]$ the inverse matrix of $\left[G_{\alpha \beta}\right]$.

Proof. Indeed, as we remarked in Section 4 , the matrix $\left[G_{\alpha \beta}\right]$ is regular. If we apply the inverse matrix $\left[G^{\alpha \beta}\right]$ to equations (6.2), then we get equations (6.3).

Equations (6.3) are the explicit form (or normal form) of the Gibbs-Appell equations (5.2).

\section{The dynamical equations of the first kind}

By setting

$$
\dot{z}^{\alpha}=\frac{d z^{\alpha}}{d t}
$$

equations (6.3) together with the constraint equations (2.1) build up a first-order differential system, in normal form, in the unknown functions $q^{i}(t)$ and $z^{\alpha}(t)$ :

$$
\begin{aligned}
& \frac{d q^{i}}{d t}=\psi^{i}(q, z), \\
& \frac{d z^{\alpha}}{d t}=G^{\alpha \beta}\left(A_{\beta}-\Gamma_{i j k} \psi^{i} \psi^{j} \psi_{\beta}^{k}\right) .
\end{aligned}
$$

Hence, we can summarize the results so far obtained in the following

Theorem 7.1. Let $Q$ be the configuration $n$-manifold of a mechanical system, with local Lagrangian coordinates $q=\left(q^{i}\right)$. Let $T Q$ be the tangent bundle of $Q$ with canonical coordinates $(q, \dot{q})=\left(q^{i}, \dot{q}^{i}\right)$. Let $C \subset T Q$ be a submanifold representing kinematical time-independent constraints. Assume that the constraint submanifold $C$ is locally described by parametric equations $\dot{q}^{i}=\psi^{i}(q, z)$ with $m<n$ parameters $z=\left(z^{\alpha}\right)$, such that the $n \times m$ matrix

$$
\left[\psi_{\alpha}^{i}\right] \doteq\left[\frac{\partial \psi^{i}}{\partial z^{\alpha}}\right]
$$

has maximal rank $m$. If the constraints are ideal, then the actual motions are represented by functions $q^{i}=q^{i}(t)$ satisfying the differential system $(7.1)^{3}$, where $\Gamma_{i j k}$ are the Christoffel symbols of the metric tensor $g_{i j}$ associated with the kinetic energy $K=\frac{1}{2} g_{i j} \dot{q}^{i} \dot{q}^{j}$ and $A_{\alpha}=A_{i} \psi_{\alpha}^{i}$, where $A_{i}$ are the Lagrangian active forces.

\footnotetext{
${ }^{3}$ Any solution of the differential system (7.1) is of course a set of functions $q^{i}(t)$ and $z^{\alpha}(t)$. But, after the integration, we can get rid of the functions $z^{\alpha}(t)$.
} 
Remark 7.1. The dynamical system (7.1) is the first-order system associated with the vector field $\mathbf{Z}$ on the constraint submanifold $C$, whose components $\left(Z^{i}, Z^{\alpha}\right)$ w.r.to the coordinates $(q, z)$ are given by

$$
\begin{aligned}
& Z^{i}=\psi^{i}(q, z), \\
& Z^{\alpha}=G^{\alpha \beta}\left(A_{\beta}-\Gamma_{i j k} \psi^{i} \psi^{j} \psi_{\beta}^{k}\right) .
\end{aligned}
$$

The $Z^{\alpha}$ are the 'vertical components' of $\mathbf{Z}$ w.r.to the projection onto $Q$. The actual motions are the projections onto $Q$ of the integral curves of $\mathbf{Z}$.

Remark 7.2. This theorem provides a first 'recipe' for writing the dynamical equations for non-holonomic systems with linear or non-linear ideal constraints:

1. Choose Lagrangian coordinates $\left(q^{i}\right)$, write the kinetic energy of the system $K=\frac{1}{2} g_{i j} \dot{q}^{i} \dot{q}^{j}$, and extract the $n \times n$ matrix $\left[g_{i j}\right]$.

2. Choose parametric equations $\dot{q}^{i}=\psi^{i}(q, z)$ of the constraints, compute the $m \times n$ matrix $\left[\psi_{\alpha}^{i}\right]$, and check its rank. If the constraints are initially expressed by implicit equations, then use (for instance) the method illustrated in Remark 7.3 below for finding parametric equations.

3. Compute the $m \times m$ matrix $\left[G_{\alpha \beta}\right]=\left[g_{i j} \psi_{\alpha}^{i} \psi_{\beta}^{j}\right]$, and the inverse matrix $\left[G^{\alpha \beta}\right]=\left[G_{\alpha \beta}\right]^{-1}$. $^{4}$

4. Write the Lagrange equations of the free motions (i.e., with only active forces $A_{i}$ ) in the form

$$
g_{i j} \ddot{q}^{j}=L_{i}(q, \dot{q})
$$

(note that the formal expression of $L_{i}$ is $L_{i}=A_{i}(q, \dot{q})-\Gamma_{h k i} \dot{q}^{h} \dot{q}^{k}$ ) and compute

$$
\bar{Z}_{i}(q, z)=L_{i}(q, \psi)=A_{i}(q, \psi)-\Gamma_{h k i}(q) \psi^{h} \psi^{k} .
$$

5. Compute $Z_{\alpha}=\bar{Z}_{i} \psi_{\alpha}^{i}$ and $Z^{\alpha}=G^{\alpha \beta} Z_{\beta}$.

6. Write the dynamical system

$$
\begin{aligned}
\frac{d q^{i}}{d t} & =\psi^{i}(q, z), \\
\frac{d z^{\alpha}}{d t} & =Z^{\alpha}(q, z) .
\end{aligned}
$$

Remark 7.3. When the constraint submanifold $C \subset T Q$ is described by a system of implicit independent equations of the kind

$$
C^{a}(q, \dot{q})=0, \quad a=1, \ldots, r, \quad r=n-m,
$$

then we have to transform these equations into parametric equations. The choice of the parameters $z^{\alpha}$ is completely free and it is only a matter of convenience, depending on the explicit concrete form of the dynamical equations (7.5) we get. Anyway, since the matrix $\left[\psi_{\alpha}^{i}\right]$ does not have the maximal rank, equations (7.6) can be solved w.r.to $m$ of the $n$ Lagrangian velocities $\dot{q}^{i}$, say - up to a reordering - w.r.to $\dot{q}^{\alpha}, \alpha=1, \ldots, m$. This process leads to considering as parameters these Lagrangian velocities: $z^{\alpha}=\dot{q}^{\alpha}$. It works very well for linear or affine constraints, where equations (7.6) have the form

$$
C_{i}^{a}(q) \dot{q}^{i}-b_{i}(q)=0 .
$$

Another possible choice of the parameters, for the linear constraints only, is that related to the use of quasi-velocities or quasi-coordinates - see for instance [9].

\footnotetext{
${ }^{4}$ Note that according to this recipe we do not have to compute the inverse $n \times n$-matrix of $\left[g_{i j}\right]$, but only the inverse of $\left[G_{\alpha \beta}\right]$, whose dimension is $m<n$.
} 
Remark 7.4. For the analysis of the qualitative (or quantitative) behavior of a non-holonomic mechanical system (like stability, equilibrium states, small oscillations, numerical integration, etc.) we can apply to $\mathbf{Z}$ all the known theorems about dynamical systems. For instance, a first integral is a function $F(q, z)$ such that

$$
\psi^{i}(q, z) \frac{\partial F}{\partial q^{i}}+Z^{\alpha}(q, z) \frac{\partial F}{\partial z^{\alpha}}=0 .
$$

Remark 7.5. The singular points of the dynamical system (7.1) are the solutions $(q, z)$ of the simultaneous equations

$$
\begin{aligned}
& \psi^{i}(q, z)=0, \\
& Z^{\alpha}(q, z) \doteq \bar{Z}_{i} \psi_{\alpha}^{i}=0 .
\end{aligned}
$$

In the case of a homogeneous quadratic constraint, $\psi^{i}(q, z)=\frac{1}{2} \psi_{\alpha \beta}^{i}(q) z^{\alpha} z^{\beta}$, equations (7.8) become

$$
\begin{aligned}
& \psi_{\alpha \beta}^{i}(q) z^{\alpha} z^{\beta}=0, \\
& \bar{Z}_{i} \psi_{\alpha \beta}^{i} z^{\beta}=0,
\end{aligned}
$$

being $\psi_{\alpha}^{i}=\psi_{\alpha \beta}^{i}(q) z^{\beta}$. This shows that, whatever $q$ and $\bar{Z}_{i}$, singular points are given by $z^{\alpha}=0$. But for $z^{\alpha}=0$ the matrix $\left[\psi_{\alpha}^{i}\right]$ does not have the maximal rank, since all its elements vanish. Hence, at these singular points the constraint $C$ is not regular.

Remark 7.6. The geometrical picture of the above results gives an intrinsic meaning of the objects we have introduced. Any vector $\mathbf{v} \in T C$ can be represented by a sum

$$
\mathbf{v}=v^{i} \partial_{i}+v^{\alpha} \partial_{\alpha}
$$

where $\partial_{i}=\partial / \partial q^{i}$ and $\partial_{\alpha}=\partial / \partial z^{\alpha}$ are interpreted as pointwise independent vector fields on $T C$ : at each point $x$ of $C$ they span the tangent space $T_{x} C$. The vectors $\partial_{\alpha}$ are 'vertical' i.e., they are tangent to the fibers $F_{q}$ of $C$. Hence, we call $v^{\alpha}$ the vertical components of $\mathbf{v}$, while we call $v^{i}$ the basic components. For instance, the basic components of $\mathbf{Z}$ are $Z^{i}=\psi^{i}(q, z)$ and the vertical components are $Z^{\alpha}$. The functions $\psi_{\alpha}^{i}$ have the role of transforming Latin components, labeled by Latin indices $h, i, j, k, \ldots$, into Greek components, labeled by Greek indices $\alpha, \beta, \ldots$.. For instance, when it is applied to a one-form (covariant vector) $\bar{Z}_{i} d q^{i}$, we get a vertical one form $Z_{\alpha} d z^{\alpha}$, and when it is applied to the covariant metric tensor $g_{i j}$, we get a metric tensor $G_{\alpha \beta}$ on the fibers of $C$, so that, by raising the indices of $Z_{\alpha}$ by the contravariant metric $G^{\alpha \beta}$, we get a vertical vector field $Z^{\alpha} \partial_{\alpha}$.

Note 7.1. I did not find equations (7.1) in the recent and old articles I have consulted. In fact, it is rather surprising that the simple idea of considering the parametric representation of the constraints as a part of the dynamical equations does not appear in the major textbooks and treatises on non-holonomic mechanics. Only recently this idea appeared in a paper of Massa and Pagani [8]. Their general approach, which is based on the jet-bundle theory and deals with timedependent constraints, leads to the introduction of the vector field $\mathbf{Z}$. The elementary approach presented here is of course quite different and leads, for instance, to different expressions of the vertical part of Z Z. Our equations (7.1) should be compared with equations (3.5b) and (3.15) of [8]. The difference is that the second equation (7.1) is written in terms of the Euclidean vectors $\mathbf{F}_{\nu}, \mathbf{v}_{\nu}$, while equation (3.15) of [8] $\mathbf{Z}$ is written in terms of the Lagrange equations, but still in an implicit form. 
Remark 7.7. For linear constraints, the approach presented here is more general than that of Čaplygin - see [9], Ch. III, $\S 3$, where the coordinates $q^{i}$ are divided into two groups, say $\left(q^{a}, q^{\alpha}\right)$, with $a=1, \ldots, m$ and $\alpha=m+1, \ldots, n$. The constraint equations are assumed to be of the form

$$
\dot{q}^{\alpha}=\sum_{a} b_{a}^{\alpha} \dot{q}^{a}
$$

where the coefficients $b_{a}^{\alpha}$ and the Lagrangian $L$ are assumed to be independent from the coordinates $\left(q^{a}\right) .^{5}$

\section{The dynamical equations of the second kind}

About the method for writing the dynamical equations of a non-holonomic mechanical system so far illustrated two remarks are in order:

- It lies on a parametric representation of the constraint $C$ (however, the vector field $\mathbf{Z}$ does not depend on the chosen parametrization).

- It does not give any information about the reactive forces.

Here, we propose an alternative method for writing the dynamical equations which is based on any implicit representation of $C$ by a system of independent equations

$$
C^{a}(q, \dot{q})=0,
$$

and which provides a way for evaluating the reactive forces.

The Lagrange equations (3.14) - see Remark 3.4 - are equivalent to the dynamical system

$$
\mathbf{X}_{\lambda}=\left\{\begin{array}{l}
\frac{d q^{i}}{d t}=\dot{q}^{i}, \\
\frac{d \dot{q}^{i}}{d t}=-\Gamma_{h k}^{i} \dot{q}^{h} \dot{q}^{k}+A^{i}+R_{\lambda}^{i},
\end{array}\right.
$$

on the tangent bundle $T Q$ of the configuration manifold $Q$. Here, $A^{i}=g^{i j} A_{j}$ and $R_{\lambda}^{i}=g^{i j} \lambda_{a} C_{j}^{a}$ are the contravariant components of the Lagrangian active and reactive forces, respectively. The label $\lambda$ points out that the reactive Lagrangian forces depend on the a priori unknown Lagrangian multipliers $\lambda=\left(\lambda_{a}\right)$, according to Theorem 3.1 and equation (3.11).

For a better understanding of what we are going to do, it is useful to consider the following

Definition 8.1. We say that a vector $\mathbf{V}$ on $T Q$ is vertical if it is tangent, at each point where it is defined, to the corresponding fiber of $T Q$. This is equivalent to say that it has the form

$$
\mathbf{V}=V^{i} \frac{\partial}{\partial \dot{q}^{i}}
$$

As a consequence, the vertical part of the vector $\mathbf{X}_{\lambda}$ is given by

$$
\left(A^{i}+R_{\lambda}^{i}-\Gamma_{h k}^{i} \dot{q}^{h} \dot{q}^{k}\right) \frac{\partial}{\partial \dot{q}^{i}}
$$

\footnotetext{
${ }^{5}$ In the history of the non-holonomic systems we can find the famous equations of Maggi, Volterra, Voronec and Čaplygin, dealing with linear constraints. The comparison of these equations with our approach is left to the reader, who can find a detailed discussion in in [9], Ch. 3. A neat illustration of the non-holonomic dynamical equations, with the essential classical and recent bibliography, can be found in the book [2].
} 
where the active and reactive forces are represented by the vertical vectors

$$
\mathbf{A}=A^{i} \frac{\partial}{\partial \dot{q}^{i}}, \quad \mathbf{R}_{\lambda}=R_{\lambda}^{i} \frac{\partial}{\partial \dot{q}^{i}},
$$

Hence, the vector $\mathbf{X}_{\lambda}$ is decomposed into the sum

$$
\mathbf{X}_{\lambda} \doteq \mathbf{X}_{G}+\mathbf{A}+\mathbf{R}_{\lambda}
$$

where $\mathbf{X}_{G}$ represents the geodesic flow,

$$
\mathbf{X}_{G}=\left\{\begin{array}{l}
\frac{d q^{i}}{d t}=\dot{q}^{i}, \\
\frac{d \dot{q}^{i}}{d t}=-\Gamma_{h k}^{i} \dot{q}^{h} \dot{q}^{k} .
\end{array}\right.
$$

We can consider $\mathbf{X}_{\lambda}=\mathbf{X}_{G}+\mathbf{A}+\mathbf{R}_{\lambda}$ as a family of vector fields, depending on the Lagrangian multipliers. However, it is a remarkable fact that we can obtain an explicit form of them, as functions of the kinematical states $(q, \dot{q})$ only.

Theorem 8.1. Let $\left[G^{a b}\right]$ be the symmetric matrix defined by

$$
G^{a b} \doteq g^{i j} C_{i}^{a} C_{j}^{b}
$$

Let $\left[G_{a b}\right]=\left[G^{a b}\right]^{-1}$ be its inverse, and $C^{a i} \doteq g^{i j} C_{j}^{a}$. If the constraints are ideal, then the Lagrangian multipliers and the Lagrangian reactive forces are well determined functions of $(q, \dot{q})$ :

$$
\begin{aligned}
& \lambda_{a}(q, \dot{q})=G_{a b}\left(C_{i}^{b}\left(\Gamma_{h k}^{i} \dot{q}^{h} \dot{q}^{k}-A^{i}\right)-\dot{q}^{i} \partial_{i} C^{b}\right), \\
& R^{i}(q, \dot{q})=G_{a b} C^{a i}\left(C_{j}^{b}\left(\Gamma_{h k}^{j} \dot{q}^{h} \dot{q}^{k}-A^{j}\right)-\dot{q}^{j} \partial_{j} C^{b}\right) .
\end{aligned}
$$

Proof. First of all, we observe that the matrix $\left[G^{a b}\right]$ is regular, since the vector fields $\mathbf{C}^{a}$ are independent. Hence, the inverse matrix $\left[G_{a b}\right]$ is well defined. In order to satisfy the constraints, the vector field $\mathbf{X}_{\lambda}$ must be tangent to the constraint submanifold $C$. This condition is expressed by equations

$$
\left\langle\mathbf{X}_{\lambda}, d C^{a}\right\rangle=0
$$

to be satisfied at least on $C$. In components, these equations read

$$
\dot{q}^{i} \partial_{i} C^{a}+\left(A^{i}-\Gamma_{h k}^{i} \dot{q}^{h} \dot{q}^{k}+R^{i}\right) C_{i}^{a}=0,
$$

i.e.,

$$
R^{i} C_{i}^{a}=C_{i}^{a}\left(\Gamma_{h k}^{i} \dot{q}^{h} \dot{q}^{k}-A^{i}\right)-\dot{q}^{i} \partial_{i} C^{a} .
$$

Note that the right hand side

$$
\Lambda^{a}(q, \dot{q}) \doteq C_{i}^{a}\left(\Gamma_{h k}^{i} \dot{q}^{h} \dot{q}^{k}-A^{i}\right)-\dot{q}^{i} \partial_{i} C^{a}
$$

is a known function of $(q, \dot{q})$. Then equation $R^{i} C_{i}^{a}=\Lambda^{a}$ assumes the form $\lambda_{b} C_{i}^{a} C_{j}^{b} g^{i j}=\Lambda^{a}$, i.e., $\lambda_{b} G^{a b}=\Lambda^{a}$. By applying the inverse matrix $\left[G_{a b}\right]$ we get equation (8.6) and equation (8.7).

The explicit form (8.7) of the reactive forces allows us to state 
Theorem 8.2. The actual motions of a mechanical system with regular and ideal non-holonomic constraints represented by a submanifold $C \subset T Q$ are the integral curves based on $C$ of the vector field

$$
\mathbf{D} \doteq \mathbf{X}_{G}+\mathbf{A}+\mathbf{R}
$$

where the components $R^{i}$ of the vertical vector $\mathbf{R}$ are defined by (8.7). If we introduce the symbols

$$
\pi^{i j} \doteq G_{a b} C^{a i} C^{b j}, \quad C^{a i} \doteq g^{i j} C_{j}^{a}, \quad C_{a}^{i} \doteq G_{a b} C^{b i}
$$

then the explicit expressions of the first-order differential system associated with $\mathbf{D}$ and of the reactive forces are

$$
\mathbf{D}=\left\{\begin{array}{l}
\frac{d q^{i}}{d t}=\dot{q}^{i}, \\
\frac{d \dot{q}^{i}}{d t}=\left(g^{i j}-\pi^{i j}\right)\left(A_{j}-\Gamma_{h k j} \dot{q}^{h} \dot{q}^{k}\right)-\dot{q}^{j} \partial_{j} C^{a} C_{a}^{i},
\end{array}\right.
$$

and

$$
R^{i}=\pi^{i j}\left(\Gamma_{h k j} \dot{q}^{h} \dot{q}^{k}-A_{j}\right)-\dot{q}^{j} \partial_{j} C^{a} C_{a}^{i},
$$

respectively.

Remark 8.1. This last theorem provides a second 'recipe' for writing the dynamical equations for non-holonomic systems with linear or non-linear ideal constraints:

1. Choose Lagrangian coordinates $\left(q^{i}\right)$, write the kinetic energy of the system $K=\frac{1}{2} g_{i j} \dot{q}^{i} \dot{q}^{j}$, extract the $n \times n$ matrix $\left[g_{i j}\right]$, and compute the inverse matrix $\left[g^{i j}\right]$.

2. Take the constraint equations $C^{a}(q, \dot{q})=0$ and compute, in the order, the following matrices:

$$
\begin{aligned}
& {\left[C_{i}^{a}\right] \doteq\left[\frac{\partial C^{a}}{\partial \dot{q}^{i}}\right] \quad \text { (seek the singular states) }} \\
& {\left[C^{a i}\right] \doteq\left[g^{i j} C_{j}^{a}\right],} \\
& {\left[G^{a b}\right]=\left[G^{b a}\right] \doteq\left[g^{i j} C_{i}^{a} C_{j}^{b}\right]=\left[C^{a i} C_{i}^{b}\right],} \\
& {\left[G_{a b}\right] \doteq\left[G^{a b}\right]^{-1},} \\
& {\left[C_{a}^{i}\right] \doteq\left[G_{a b} C^{b i}\right],} \\
& {\left[\pi^{i j}\right]=\left[\pi^{j i}\right] \doteq\left[C^{a i} C_{a}^{j}\right],} \\
& {\left[g^{i j}-\pi^{i j}\right],} \\
& {\left[\partial_{i} C^{a}\right] \doteq\left[\frac{\partial C^{a}}{\partial q^{i}}\right]} \\
& {\left[\partial_{j} C^{a} C_{a}^{i}\right] .}
\end{aligned}
$$

3. Write the Lagrange equations for the free motions in the form

$$
g_{i j} \ddot{q}^{j}=A_{i}-\Gamma_{h k i} \dot{q}^{h} \dot{q}^{k},
$$

and keep in evidence the functions

$$
L_{i}(q, \dot{q}) \doteq A_{i}-\Gamma_{h k i} \dot{q}^{h} \dot{q}^{k} .
$$


4. Compute the vector

$$
D^{i} \doteq\left(g^{i j}-\pi^{i j}\right) L_{j}-\dot{q}^{j} \partial_{j} C^{a} C_{a}^{i}
$$

5. Write the differential system (8.12),

$$
\mathbf{D}=\left\{\begin{array}{l}
\frac{d q^{i}}{d t}=\dot{q}^{i}, \\
\frac{d \dot{q}^{i}}{d t}=D^{i}
\end{array}\right.
$$

6. Its solutions $q^{i}(t), \dot{q}^{i}(t)$, with initial conditions belonging to $C$, describe the actual motions of the system.

7. Evaluate the reactive forces along any actual motion by means of equation (8.13),

$$
R^{i}=-\pi^{i j} L_{j}-\dot{q}^{j} \partial_{j} C^{a} C_{a}^{i} .
$$

Remark 8.2. The case of single constraint equation $C(q, \dot{q})=0$. In this case the abovegiven recipe can be applied by setting $a=b=1$. Items 1 and 2 of the general recipe still hold. However, since some of the above matrices reduces to scalar functions or to vectors, the index 1 can be omitted or replaced by $*$ :

$$
\begin{aligned}
& {\left[C_{i}\right] \doteq\left[\frac{\partial C}{\partial \dot{q}^{i}}\right],} \\
& {\left[C^{i}\right] \doteq\left[g^{i j} C_{j}\right],} \\
& G \doteq\left[g^{i j} C_{i} C_{j}\right]=C^{i} C_{i}, \\
& C_{*}^{i} \doteq G^{-1} C^{i}, \\
& {\left[\pi^{i j}\right] \doteq\left[C^{i} C_{*}^{j}\right]=G^{-1}\left[C^{i} C^{j}\right],} \\
& {\left[g^{i j}-\pi^{i j}\right],} \\
& \partial_{i} C \doteq \frac{\partial C}{\partial q^{i}}, \\
& {\left[\partial_{j} C C_{*}^{i}\right] .}
\end{aligned}
$$

Then, follows items $3-7$ of the general recipe.

\section{Illustrative examples}

As shown above, for writing the dynamical equations of a non-holonomic system we can apply two methods: the first method is established by Theorem 7.1 and the corresponding recipe is illustrated in Remark 7.2; the second method is established by Theorem 8.2 and the corresponding recipe is illustrated in Remark 8.1.

Let us see how these two methods work by concrete examples. We begin with two paradigmatic and simple examples of linear non-holonomic constraints, the 'skate' and the 'vertical rolling disc'. Then, we shall consider two more demanding examples: 'two co-axial rolling discs' and 'two points with parallel velocities'. This last one is a genuine non-linear non-holonomic system.

In illustrating examples of application of a theory it is not customary, in general, to provide detailed calculations - which usually are left to the reader. Here, however, it is worthwhile to disregard such a custom in order to compare the effectiveness of the two methods (mainly the length of the calculations) applied to a same mechanical system. 


\subsection{The skate}

This mechanical system is made of a homogeneous rod (material segment) sliding without friction on a plane ${ }^{6}$. The configurations of the skate are determined by the Cartesian coordinates $(x, y)$ of the center of mass (i.e., of the segment) $G$ and by the angle $\theta$ of the rod w.r.to the $x$ axis. The configuration manifold $Q$ is $\mathbb{R} \times \mathbb{S}_{1}$ and natural ordered Lagrangian coordinates are $\left(q^{1}, q^{2}, q^{3}\right)=(x, y, \theta)$. The velocity $\mathbf{v}_{G}=[\dot{x}, \dot{y}]$ of the mass-center is constrained to be parallel to the rod. This constraint is then represented by a single equation:

$$
\dot{x} \sin \theta-\dot{y} \cos \theta=0 \text {. }
$$

The kinetic energy is given by $K=\frac{1}{2} m\left(\dot{x}^{2}+\dot{y}^{2}\right)+\frac{1}{2} I \dot{\theta}$, where $m$ and $I$ are the mass and the moment of inertia w.r.to $G$ (i.e., w.r.to the line orthogonal to the plane through $G$ ), respectively.

(i) First method. Since $\operatorname{dim}(T Q)=6$ and $\operatorname{dim}(C)=3$, we need two parameters $\left(z^{1}, z^{2}\right)$ for describing $C$. We can consider the parametric equations

$$
\dot{x}=z^{1} \cos \theta, \quad \dot{y}=z^{1} \sin \theta, \quad \dot{\theta}=z^{2} .
$$

Then, we compute the necessary matrices and vectors:

$$
\begin{array}{ll}
{\left[g_{i j}\right]} & =\left[\begin{array}{ccc}
m & 0 & 0 \\
0 & m & 0 \\
0 & 0 & I
\end{array}\right], \\
{\left[\psi^{i}\right]} & =\left[z^{1} \cos \theta, z^{1} \sin \theta, z^{2}\right], \quad\left[\psi_{\alpha}^{i}\right]=\left[\begin{array}{ccc}
\cos \theta & \sin \theta & 0 \\
0 & 0 & 1
\end{array}\right] .
\end{array}
$$

This matrix has maximal rank everywhere: the constraint is regular.

$$
\left[G_{\alpha \beta}\right]=\left[\begin{array}{cc}
m & 0 \\
0 & I
\end{array}\right], \quad\left[G^{\alpha \beta}\right]=\left[\begin{array}{cc}
\frac{1}{m} & 0 \\
0 & \frac{1}{I}
\end{array}\right]
$$

Since,

$$
\left[\frac{\partial K}{\partial \dot{q}^{i}}\right]=[m \dot{x}, m \dot{y}, I \dot{\theta}], \quad\left[\frac{\partial K}{\partial q^{i}}\right]=[0,0,0],
$$

the Lagrange equations for the free motions $g_{i j} \ddot{q}^{j}=A_{i}-\Gamma_{h k i} \dot{q}^{h} \dot{q}^{k} \mathrm{read}$

$$
m \ddot{x}=A_{1}, \quad m \ddot{y}=A_{2}, \quad I \ddot{\theta}=A_{3} .
$$

Hence, $L_{i}=A_{i}(q, \dot{q}), \bar{Z}_{i}=A_{i}(q, \psi)$ and

$$
\left[Z_{\alpha}\right]=\left[\psi_{\alpha}^{i} \bar{Z}_{i}\right]=\left[\begin{array}{c}
A_{1} \cos \theta+A_{2} \sin \theta \\
A_{3}
\end{array}\right] .
$$

The dynamical equations are

$$
\begin{array}{rlrl}
\frac{d x}{d t} & =z^{1} \cos \theta, & \frac{d z^{1}}{d t} & =\frac{1}{m}\left(A_{1} \cos \theta+A_{2} \sin \theta\right), \\
\frac{d y}{d t} & =z^{1} \sin \theta, & \frac{d z^{2}}{d t} & =\frac{A_{3}}{I} . \\
\frac{d \theta}{d t} & =z^{2}, &
\end{array}
$$

\footnotetext{
${ }^{6}$ Quite similar classical examples are that of two material points linked by a massless rigid segment [5], p. 23 and 63, and the Čaplygin sleigh [9], Ch. III, § 3, Examples $2 \& 5$, and Ch. V, § 4. Another example of this kind is examined in [4], $\S 4.1 \& \S 4.2$.
} 
(ii) Second method, for a single constraint equation - Remark 8.2: $C \doteq \dot{x} \sin \theta-\dot{y} \cos \theta=0$.

$$
\begin{aligned}
& {\left[g^{i j}\right]=\left[\begin{array}{ccc}
\frac{1}{m} & 0 & 0 \\
0 & \frac{1}{m} & 0 \\
0 & 0 & \frac{1}{I}
\end{array}\right]} \\
& {\left[C_{i}\right] \doteq\left[\frac{\partial C}{\partial \dot{q}^{i}}\right]=[\sin \theta,-\cos \theta, 0],} \\
& {\left[C^{i}\right] \doteq\left[g^{i j} C_{j}\right]=\left[\frac{\sin \theta}{m},-\frac{\cos \theta}{m}, 0\right],} \\
& G \doteq C^{i} C_{i}=\frac{1}{m} \\
& {\left[C_{*}^{i}\right] \doteq G^{-1}\left[C^{i}\right]=[\sin \theta,-\cos \theta, 0]=\left[C_{i}\right],} \\
& {\left[\pi^{i j}\right] \doteq G^{-1}\left[C^{i} C^{j}\right]=\frac{1}{m}\left[\begin{array}{ccc}
\sin ^{2} \theta & -\sin \theta \cos \theta & 0 \\
-\sin \theta \cos \theta & \cos ^{2} \theta & 0 \\
0 & 0 & 0
\end{array}\right] \text {, }} \\
& {\left[g^{i j}-\pi^{i j}\right]=\frac{1}{m}\left[\begin{array}{ccc}
\cos ^{2} \theta & \sin \theta \cos \theta & 0 \\
\sin \theta \cos \theta & \sin ^{2} \theta & 0 \\
0 & 0 & \frac{m}{I}
\end{array}\right],} \\
& {\left[\partial_{i} C\right] \doteq\left[\frac{\partial C}{\partial q^{i}}\right]=[0,0, \dot{x} \cos \theta+\dot{y} \sin \theta] \text {, }} \\
& {\left[\dot{q}^{j} \partial_{j} C C_{*}^{i}\right]=\dot{\theta}(\dot{x} \cos \theta+\dot{y} \sin \theta)\left[C_{*}^{i}\right] .}
\end{aligned}
$$

Since

$$
\left[\frac{\partial K}{\partial \dot{q}^{i}}\right]=[m \dot{x}, m \dot{y}, I \dot{\theta}], \quad\left[\frac{\partial K}{\partial q^{i}}\right]=[0,0,0],
$$

the Lagrange equations read

$$
m \ddot{x}=A_{1}, \quad m \ddot{y}=A_{2}, \quad I \ddot{\theta}=A_{3} .
$$

Hence, $L_{i}=A_{i}$. We have all the ingredients for computing the vector $D^{i} \doteq\left(g^{i j}-\pi^{i j}\right) L_{j}-$ $\dot{q}^{j} \partial_{j} C C_{*}^{i}:$

$$
\begin{aligned}
D^{1} & =\frac{\cos \theta}{m}\left(A_{1} \cos \theta+A_{2} \sin \theta\right)-\dot{\theta}(\dot{x} \cos \theta+\dot{y} \sin \theta) \sin \theta, \\
D^{2} & =\frac{\sin \theta}{m}\left(A_{1} \cos \theta+A_{2} \sin \theta\right)+\dot{\theta}(\dot{x} \cos \theta+\dot{y} \sin \theta) \cos \theta, \\
D^{3} & =\frac{1}{I} A_{3},
\end{aligned}
$$

and the differential system (8.12) reads

$$
\begin{array}{ll}
\frac{d x}{d t}=\dot{x}, & \frac{d \dot{x}}{d t}=\frac{\cos \theta}{m}\left(A_{1} \cos \theta+A_{2} \sin \theta\right)-\dot{\theta}(\dot{x} \cos \theta+\dot{y} \sin \theta) \sin \theta, \\
\frac{d y}{d t}=\dot{y}, & \frac{d \dot{y}}{d t}=\frac{\sin \theta}{m}\left(A_{1} \cos \theta+A_{2} \sin \theta\right)+\dot{\theta}(\dot{x} \cos \theta+\dot{y} \sin \theta) \cos \theta, \\
\frac{d \theta}{d t}=\dot{\theta}, & \frac{d \dot{\theta}}{d t}=I^{-1} A_{3} .
\end{array}
$$




\subsection{The vertical rolling disc}

A material disc of radius $R$ running on a plane is kept perpendicular to it by massless and frictionless devices. The configuration manifold is $Q_{4}=\mathbb{R}^{2} \times \mathbb{S}_{1} \times \mathbb{S}_{1}$, with coordinates $\left(q^{1}, q^{2}, q^{3}, q^{4}\right)=(x, y, \theta, \psi)$, where $(x, y)$ are Cartesian coordinates of the center $P$ of the disc (i.e., of the point $C$ in contact with the plane), $\theta$ a rotation angle of the disk around its axis, and $\psi$ an angle giving the orientation of the axis (see Figure 4, with $\theta=\theta_{1}$ ). Constraint: the disc rolls on the plane without sliding. Let $(\mathbf{i}, \mathbf{j}, \mathbf{k})$ be the unitary vectors associated with the $(x, y, z)$-axes. The unitary vector $\mathbf{k}$ is associated with the oriented angle $\psi$. Let $\mathbf{u}$ be the unitary vector associated with the oriented angle $\theta$. Then, $\mathbf{u}=\cos \psi \mathbf{j}-\sin \psi \mathbf{i}$. The angular velocity $\omega$ is given by $\omega=\dot{\theta} \mathbf{u}+\dot{\psi} \mathbf{k}$. The velocity $\mathbf{v}_{C}$ of the point $C$ is given by $\mathbf{v}_{C}=\mathbf{v}_{P}+\omega \times P C$, where $P C=-R \mathbf{k}$. Hence,

$$
\mathbf{v}_{C}=\dot{x} \mathbf{i}+\dot{y} \mathbf{j}-(\dot{\theta} \mathbf{u}+\dot{\psi} \mathbf{k}) \times R \mathbf{k}=\dot{x} \mathbf{i}+\dot{y} \mathbf{j}-R \dot{\theta} \mathbf{u} \times \mathbf{k}
$$

Since $\mathbf{u} \times \mathbf{k}=\cos \psi \mathbf{j} \times \mathbf{k}-\sin \psi \mathbf{i} \times \mathbf{k}$, we get

$$
\begin{aligned}
\mathbf{v}_{C} & =\dot{x} \mathbf{i}+\dot{y} \mathbf{j}-R \dot{\theta}(\cos \psi \mathbf{j} \times \mathbf{k}-\sin \psi \mathbf{i} \times \mathbf{k})=\dot{x} \mathbf{i}+\dot{y} \mathbf{j}-R \dot{\theta}(\cos \psi \mathbf{i}+\sin \psi \mathbf{j}) \\
& =(\dot{x}-R \dot{\theta} \cos \psi) \mathbf{i}+(\dot{y}-R \dot{\theta} \sin \psi) \mathbf{j} .
\end{aligned}
$$

The kinematical constraint $\mathbf{v}_{C}=0$ is then represented by the following two linear equations

$$
\begin{aligned}
& C^{1} \doteq \dot{x}-R \cos \psi \dot{\theta}=0, \\
& C^{2} \doteq \dot{y}-R \sin \psi \dot{\theta}=0 .
\end{aligned}
$$

(i) First method. Assume that the center of mass of the disc coincides with its geometrical center. Then the kinetic energy is given by

$$
K=\frac{1}{2} m\left(\dot{x}^{2}+\dot{y}^{2}\right)+\frac{1}{2}\left(A \dot{\theta}^{2}+B \dot{\psi}^{2}\right),
$$

where $m$ is the mass, $A$ and $B$ are the moments of inertia w.r.to the axis of rotation and a diameter, respectively. Thus,

$$
\left[g_{i j}\right]=\left[\begin{array}{cccc}
m & 0 & 0 & 0 \\
0 & m & 0 & 0 \\
0 & 0 & A & 0 \\
0 & 0 & 0 & B
\end{array}\right]
$$

From the constraint equations (9.2) we get the parametric equations

$$
\begin{array}{ll}
\dot{x}=R \cos \psi z^{1}, & \dot{\theta}=z^{1}, \\
\dot{y}=R \sin \psi z^{1}, & \dot{\psi}=z^{2} .
\end{array}
$$

Thus,

$$
\begin{aligned}
& {\left[\psi^{i}\right]=\left[R \cos \psi z^{1}, R \sin \psi z^{1}, z^{1}, z^{2}\right],} \\
& {\left[\psi_{\alpha}^{i}\right]=\left[\begin{array}{cccc}
R \cos \psi & R \sin \psi & 1 & 0 \\
0 & 0 & 0 & 1
\end{array}\right] \quad(\alpha=1,2, \text { index of line }) .}
\end{aligned}
$$

This matrix has maximal rank, thus the constraint is regular. It follows that

$$
\begin{aligned}
& G_{\alpha \beta}=g_{i j} \psi_{\alpha}^{i} \psi_{\beta}^{j}=m \psi_{\alpha}^{1} \psi_{\beta}^{1}+m \psi_{\alpha}^{2} \psi_{\beta}^{2}+A \psi_{\alpha}^{3} \psi_{\beta}^{3}+B \psi_{\alpha}^{4} \psi_{\beta}^{4}, \\
& G_{11}=m \psi_{1}^{1} \psi_{1}^{1}+m \psi_{1}^{2} \psi_{1}^{2}+A \psi_{1}^{3} \psi_{1}^{3}+B \psi_{1}^{4} \psi_{1}^{4}=m R^{2} \cos ^{2} \psi+m R^{2} \sin ^{2} \psi+A=m R^{2}+A,
\end{aligned}
$$




$$
\begin{aligned}
& G_{12}=m \psi_{1}^{1} \psi_{2}^{1}+m \psi_{1}^{2} \psi_{2}^{2}+A \psi_{1}^{3} \psi_{2}^{3}+B \psi_{1}^{4} \psi_{2}^{4}=0, \\
& G_{22}=m \psi_{2}^{1} \psi_{2}^{1}+m \psi_{2}^{2} \psi_{2}^{2}+A \psi_{2}^{3} \psi_{2}^{3}+B \psi_{2}^{4} \psi_{2}^{4}=B, \\
& {\left[G_{\alpha \beta}\right]=\left[\begin{array}{cc}
m R^{2}+A & 0 \\
0 & B
\end{array}\right],} \\
& {\left[G^{\alpha \beta}\right]=\left[\begin{array}{cc}
\frac{1}{m R^{2}+A} & 0 \\
0 & \frac{1}{B}
\end{array}\right] .}
\end{aligned}
$$

Since,

$$
\left[\frac{\partial K}{\partial \dot{q}^{i}}\right]=[m \dot{x}, m \dot{y}, A \dot{\theta}, B \dot{\psi}], \quad\left[\frac{\partial K}{\partial q^{i}}\right]=[0,0,0,0],
$$

the Lagrange equations for the free motions $g_{i j} \ddot{q}^{j}=A_{i}-\Gamma_{h k i} \dot{q}^{h} \dot{q}^{k}$ read

$$
m \ddot{x}=A_{1}, \quad m \ddot{y}=A_{2}, \quad A \ddot{\theta}=A_{3}, \quad B \ddot{\psi}=A_{4} .
$$

This shows that $L_{i}=A_{i}$. Hence, $\bar{Z}_{i}=A_{i}(q, \psi)$, and

$$
\begin{aligned}
& {\left[Z_{\alpha}\right]=\left[\bar{Z}_{i} \psi_{\alpha}^{i}\right]=\left[\begin{array}{c}
A_{1} R \cos \psi+A_{2} R \sin \psi+A_{3} \\
A_{4}
\end{array}\right],} \\
& {\left[Z^{\alpha}\right]=\left[G^{\alpha \beta} Z_{\beta}\right]=\left[\begin{array}{c}
\frac{A_{1} R \cos \psi+A_{2} R \sin \psi+A_{3}}{m R^{2}+A} \\
\frac{A_{4}}{B}
\end{array}\right] .}
\end{aligned}
$$

Thus, the dynamical equations are

$$
\begin{array}{llll}
\frac{d x}{d t} & =R \cos \psi z^{1}, & & \\
\frac{d y}{d t} & =R \sin \psi z^{1}, & \frac{d z^{1}}{d t}=\left(m R^{2}+A\right)^{-1}\left(A_{1} R \cos \psi+A_{2} R \sin \psi+A_{3}\right), \\
\frac{d \theta}{d t}=z^{1}, & \frac{d z^{2}}{d t}=B^{-1} A_{4} . & \\
\frac{d \psi}{d t}=z^{2}, & &
\end{array}
$$

(ii) Second method. Recall the constraint equations (9.2). Then,

$$
\begin{aligned}
& {\left[C_{i}^{a}\right] \doteq\left[\frac{\partial C^{a}}{\partial \dot{q}^{i}}\right]=\left[\begin{array}{cccc}
1 & 0 & -R \cos \psi & 0 \\
0 & 1 & -R \sin \psi & 0
\end{array}\right],} \\
& {\left[C^{a i}\right] \doteq\left[g^{i j} C_{j}^{a}\right]=\left[\begin{array}{cccc}
\frac{1}{m} & 0 & -\frac{R}{A} \cos \psi & 0 \\
0 & \frac{1}{m} & -\frac{R}{A} \sin \psi & 0
\end{array}\right] \text {, }} \\
& {\left[G^{a b}\right] \doteq\left[C^{a i} C_{i}^{b}\right]=\left[\begin{array}{cc}
\frac{1}{m}+\frac{R^{2}}{A} \cos ^{2} \psi & \frac{R^{2}}{A} \sin \psi \cos \psi \\
\frac{R^{2}}{A} \sin \psi \cos \psi & \frac{1}{m}+\frac{R^{2}}{A} \sin ^{2} \psi
\end{array}\right] \text {, }} \\
& G \doteq \operatorname{det}\left[G^{a b}\right]=\frac{1}{m^{2}}+\frac{R^{2}}{m A}, \quad G^{-1}=\frac{m^{2} A}{m R^{2}+A}, \\
& {\left[G_{a b}\right]=G^{-1}\left[\begin{array}{cc}
\frac{1}{m}+\frac{R^{2}}{A} \sin ^{2} \psi & -\frac{R^{2}}{A} \sin \psi \cos \psi \\
-\frac{R^{2}}{A} \sin \psi \cos \psi & \frac{1}{m}+\frac{R^{2}}{A} \cos ^{2} \psi
\end{array}\right],}
\end{aligned}
$$




$$
\left[C_{a}^{i}\right]=\left[G_{a b} C^{b i}\right]=G^{-1}\left[\begin{array}{cccc}
\frac{1}{m}\left(\frac{1}{m}+\frac{R^{2}}{A} \sin ^{2} \psi\right) & -\frac{R^{2}}{m A} \sin \psi \cos \psi & * C_{1}^{3} & 0 \\
-\frac{R^{2}}{m A} \sin \psi \cos \psi & \frac{1}{m}\left(\frac{1}{m}+\frac{R^{2}}{A} \cos ^{2} \psi\right) & * C_{2}^{3} & 0
\end{array}\right],
$$

where

$$
\begin{aligned}
* C_{1}^{3} & =\left(\frac{1}{m}+\frac{R^{2}}{A} \sin ^{2} \psi\right)\left(-\frac{R}{A} \cos \psi\right)+\left(-\frac{R^{2}}{A} \sin \psi \cos \psi\right)\left(-\frac{R}{A} \sin \psi\right) \\
& =-\frac{R}{m A} \cos \psi-\frac{R^{3}}{A^{2}} \sin ^{2} \psi \cos \psi+\frac{R^{3}}{A^{2}} \sin ^{2} \psi \cos \psi=-\frac{R}{m A} \cos \psi, \\
* C_{2}^{3} & =\left(-\frac{R^{2}}{A} \sin \psi \cos \psi\right)\left(-\frac{R}{A} \cos \psi\right)+\left(\frac{1}{m}+\frac{R^{2}}{A} \cos ^{2} \psi\right)\left(-\frac{R}{A} \sin \psi\right) \\
& =\left(-\frac{R^{2}}{A} \sin \psi \cos \psi\right)\left(-\frac{R}{A} \cos \psi\right)+\left(\frac{1}{m}+\frac{R^{2}}{A} \cos ^{2} \psi\right)\left(-\frac{R}{A} \sin \psi\right) \\
& =-\frac{R}{m A} \sin \psi .
\end{aligned}
$$

Hence,

$$
\left[C_{a}^{i}\right]=G^{-1}\left[\begin{array}{cccc}
\frac{1}{m}\left(\frac{1}{m}+\frac{R^{2}}{A} \sin ^{2} \psi\right) & -\frac{R^{2}}{m A} \sin \psi \cos \psi & -\frac{R}{m A} \cos \psi & 0 \\
-\frac{R^{2}}{m A} \sin \psi \cos \psi & \frac{1}{m}\left(\frac{1}{m}+\frac{R^{2}}{A} \cos ^{2} \psi\right) & -\frac{R}{m A} \sin \psi & 0
\end{array}\right] .
$$

Let us compute $\pi^{i j} \doteq C^{a i} C_{a}^{j}=\pi^{j i}$. Let us set $* \pi^{i j} \doteq G \pi^{i j}$. Then,

$$
\left[\pi^{i j}\right]=G^{-1}\left[\begin{array}{cccc}
\frac{1}{m^{2}}\left(\frac{1}{m}+\frac{R^{2}}{A} \sin ^{2} \psi\right) & -\frac{R^{2}}{m^{2} A} \sin \psi \cos \psi & * \pi^{14} & 0 \\
-\frac{R^{2}}{m^{2} A} \sin \psi \cos \psi & \frac{1}{m^{2}}\left(\frac{1}{m}+\frac{R^{2}}{A} \cos ^{2} \psi\right) & * \pi^{24} & 0 \\
* \pi^{31} & * \pi^{32} & * \pi^{33} & 0 \\
* \pi^{41} & * \pi^{42} & 0 & 0
\end{array}\right],
$$

where

$$
\begin{aligned}
* \pi^{13} & =\frac{1}{m}\left(\frac{1}{m}+\frac{R^{2}}{A} \sin ^{2} \psi\right)\left(-\frac{R}{A} \cos \psi\right)+\left(-\frac{R^{2}}{m A} \sin \psi \cos \psi\right)\left(-\frac{R}{A} \sin \psi\right) \\
& =\frac{1}{m}\left(\frac{1}{m}+\frac{R^{2}}{A} \sin ^{2} \psi\right)\left(-\frac{R}{A} \cos \psi\right)+\frac{R^{3}}{m A^{2}} \sin ^{2} \psi \cos \psi=-\frac{R}{m^{2} A} \cos \psi, \\
* \pi^{23} & =\left(-\frac{R^{2}}{m A} \sin \psi \cos \psi\right)\left(-\frac{R}{A} \cos \psi\right)+\frac{1}{m}\left(\frac{1}{m}+\frac{R^{2}}{A} \cos ^{2} \psi\right)\left(-\frac{R}{A} \sin \psi\right) \\
& =-\frac{R}{m^{2} A} \sin \psi .
\end{aligned}
$$

It follows that

$$
\left[\pi^{i j}\right]=G^{-1}\left[\begin{array}{cccc}
\frac{1}{m^{2}}\left(\frac{1}{m}+\frac{R^{2}}{A} \sin ^{2} \psi\right) & -\frac{R^{2}}{m^{2} A} \sin \psi \cos \psi & -\frac{R}{m^{2} A} \cos \psi & 0 \\
-\frac{R^{2}}{m^{2} A} \sin \psi \cos \psi & \frac{1}{m^{2}}\left(\frac{1}{m}+\frac{R^{2}}{A} \cos ^{2} \psi\right) & -\frac{R}{m^{2} A} \sin \psi & 0 \\
-\frac{R}{m^{2} A} \cos \psi & -\frac{R}{m^{2} A} \sin \psi & \frac{R^{2}}{m A^{2}} & 0 \\
0 & 0 & 0 & 0
\end{array}\right] \text {, }
$$




$$
\begin{aligned}
& {\left[g^{i j}-\pi^{i j}\right]=G^{-1}\left[\begin{array}{cccc}
\frac{G}{m}-\frac{1}{m^{2}}\left(\frac{1}{m}+\frac{R^{2}}{A} \sin ^{2} \psi\right) & \frac{R^{2}}{m^{2} A} \sin \psi \cos \psi & \frac{R}{m^{2} A} \cos \psi & 0 \\
\frac{R^{2}}{m^{2} A} \sin \psi \cos \psi & \frac{G}{m}-\frac{1}{m^{2}}\left(\frac{1}{m}+\frac{R^{2}}{A} \cos ^{2} \psi\right) & \frac{R}{m^{2} A} \sin \psi & 0 \\
\frac{R}{m^{2} A} \cos \psi & \frac{R}{m^{2} A} \sin \psi & \frac{G}{A}-\frac{R^{2}}{m A^{2}} & 0 \\
0 & 0 & 0 & \frac{G}{B}
\end{array}\right]} \\
& =\frac{m^{2} A}{m R^{2}+A}\left[\begin{array}{cccc}
\frac{G}{m}-\frac{1}{m^{2}}\left(\frac{1}{m}+\frac{R^{2}}{A} \sin ^{2} \psi\right) & \frac{R^{2}}{m^{2} A} \sin \psi \cos \psi & \frac{R}{m^{2} A} \cos \psi & 0 \\
\frac{R^{2}}{m^{2} A} \sin \psi \cos \psi & \frac{G}{m}-\frac{1}{m^{2}}\left(\frac{1}{m}+\frac{R^{2}}{A} \cos ^{2} \psi\right) & \frac{R}{m^{2} A} \sin \psi & 0 \\
\frac{R}{m^{2} A} \cos \psi & \frac{R}{m^{2} A} \sin \psi & \frac{G}{A}-\frac{R^{2}}{m A^{2}} & 0 \\
0 & 0 & 0 & \frac{G}{B}
\end{array}\right] \\
& =\frac{1}{m R^{2}+A}\left[\begin{array}{cccc}
R^{2} \cos ^{2} \psi & R^{2} \sin \psi \cos \psi & R \cos \psi & 0 \\
R^{2} \sin \psi \cos \psi & R^{2} \sin ^{2} \psi & R \sin \psi & 0 \\
R \cos \psi & R \sin \psi & 1 & 0 \\
0 & 0 & 0 & \frac{m R^{2}+A}{B}
\end{array}\right] \text {. }
\end{aligned}
$$

Recall once more equations (9.2). Then,

$$
\left[\partial_{i} C^{a}\right] \doteq\left[\frac{\partial C^{a}}{\partial q^{i}}\right]\left[\begin{array}{cccc}
0 & 0 & 0 & R \sin \psi \dot{\theta} \\
0 & 0 & 0 & -R \cos \psi \dot{\theta}
\end{array}\right], \quad\left[\dot{q}^{i} \partial_{i} C^{a}\right]=\left[\begin{array}{c}
R \sin \psi \dot{\theta} \dot{\psi} \\
-R \cos \psi \dot{\theta} \dot{\psi}
\end{array}\right]
$$

Let us set $X^{i} \doteq \dot{q}^{j} \partial_{j} C^{a} C_{a}^{i}$. Then,

$$
\begin{aligned}
G X^{1} & =(R \sin \psi \dot{\theta} \dot{\psi})\left(\frac{1}{m}\left(\frac{1}{m}+\frac{R^{2}}{A} \sin ^{2} \psi\right)\right)+(-R \cos \psi \dot{\theta} \dot{\psi})\left(-\frac{R^{2}}{m A} \sin \psi \cos \psi\right) \\
& =\frac{R}{m^{2}} \sin \psi \dot{\theta} \dot{\psi}+\frac{R^{3}}{m A} \sin \psi \dot{\theta} \dot{\psi}=\frac{R}{m^{2}} \sin \psi \dot{\theta} \dot{\psi}\left(1+\frac{m R^{2}}{A}\right) \\
& =\frac{R\left(m R^{2}+A\right)}{m^{2} A} \sin \psi \dot{\theta} \dot{\psi}, \\
G X^{2} & =(R \sin \psi \dot{\theta} \dot{\psi})\left(-\frac{R^{2}}{m A} \sin \psi \cos \psi\right)+(-R \cos \psi \dot{\theta} \dot{\psi})\left(\frac{1}{m}\left(\frac{1}{m}+\frac{R^{2}}{A} \cos ^{2} \psi\right)\right) \\
& =-\frac{R}{m^{2}} \cos \psi \dot{\theta} \dot{\psi}-\frac{R^{3}}{m A} \cos \psi \dot{\theta} \dot{\psi}=-\frac{R\left(m R^{2}+A\right)}{m^{2} A} \cos \psi \dot{\theta} \dot{\psi}, \\
G X^{3} & =(R \sin \psi \dot{\theta} \dot{\psi})\left(-\frac{R}{m A} \cos \psi\right)+(-R \cos \psi \dot{\theta} \dot{\psi})\left(-\frac{R}{m A} \sin \psi\right)=0, \\
G X^{4} & =(R \sin \psi \dot{\theta} \dot{\psi})(0)+(-R \cos \psi \dot{\theta} \dot{\psi})(0)=0 .
\end{aligned}
$$

Since $G^{-1}=\frac{m^{2} A}{m R^{2}+A}$, we get

$$
\begin{aligned}
{\left[X^{i}\right] } & =\left[\dot{q}^{j} \partial_{j} C^{a} C_{a}^{i}\right]=G^{-1}\left[\frac{R\left(m R^{2}+A\right)}{m^{2} A} \sin \psi \dot{\theta} \dot{\psi},-\frac{R\left(m R^{2}+A\right)}{m^{2} A} \cos \psi \dot{\theta} \dot{\psi}, 0,0\right] \\
& =R \dot{\theta} \dot{\psi}[\sin \psi,-\cos \psi, 0,0] .
\end{aligned}
$$


Now we are able to compute the components $D^{i} \doteq\left(g^{i j}-\pi^{i j}\right) L_{j}-\dot{q}^{j} \partial_{j} C^{a} C_{a}^{i}$ of the vector $\mathbf{D}$ :

$$
\begin{aligned}
D^{1} & =\frac{1}{m R^{2}+A}\left(A_{1} R^{2} \cos ^{2} \psi+A_{2} R^{2} \sin \psi \cos \psi+A_{3} R \cos \psi\right)-R \dot{\theta} \dot{\psi} \sin \psi \\
& =\frac{R \cos \psi}{m R^{2}+A}\left(A_{1} R \cos \psi+A_{2} R \sin \psi+A_{3}\right)-R \dot{\theta} \dot{\psi} \sin \psi, \\
D^{2} & =\frac{1}{m R^{2}+A}\left(A_{1} R^{2} \sin \psi \cos \psi+A_{2} R^{2} \sin ^{2} \psi+A_{3} R \sin \psi\right)+R \dot{\theta} \dot{\psi} \cos \psi \\
& =\frac{R \sin \psi}{m R^{2}+A}\left(A_{1} R \cos \psi+A_{2} R \sin \psi+A_{3}\right)+R \dot{\theta} \dot{\psi} \cos \psi, \\
D^{3} & =\frac{1}{m R^{2}+A}\left(A_{1} R \cos \psi+A_{2} R \sin \psi+A_{3}\right) \\
& =\frac{1}{m R^{2}+A}\left(A_{1} R \cos \psi+A_{2} R \sin \psi+A_{3}\right), \\
D^{4} & =\frac{A_{4}}{B}
\end{aligned}
$$

The resulting dynamical system (8.12) is

$$
\begin{array}{lll}
\frac{d x}{d t}=\dot{x}, & \frac{d \dot{x}}{d t}=\frac{R \cos \psi}{m R^{2}+A}\left(A_{1} R \cos \psi+A_{2} R \sin \psi+A_{3}\right)-R \dot{\theta} \dot{\psi} \sin \psi, \\
\frac{d y}{d t}=\dot{y}, & \frac{d \dot{y}}{d t}=\frac{R \sin \psi}{m R^{2}+A}\left(A_{1} R \cos \psi+A_{2} R \sin \psi+A_{3}\right)+R \dot{\theta} \dot{\psi} \cos \psi, \\
\frac{d \theta}{d t}=\dot{\theta}, & \frac{d \dot{\theta}}{d t}=\frac{1}{m R^{2}+A}\left(A_{1} R \cos \psi+A_{2} R \sin \psi+A_{3}\right), \\
\frac{d \psi}{d t}=\dot{\psi}, & \frac{d \dot{\psi}}{d t}=\frac{A_{4}}{B}
\end{array}
$$

By introducing the new variables

$$
X \doteq \frac{m R^{2}+A}{R} x, \quad Y \doteq \frac{m R^{2}+A}{R} y, \quad \Theta \doteq\left(m R^{2}+A\right) \theta,
$$

it assumes the more compact form

$$
\begin{array}{rlrl}
\frac{d X}{d t} & =\dot{X}, & & \frac{d \dot{X}}{d t}=\cos \psi\left(A_{1} R \cos \psi+A_{2} R \sin \psi+A_{3}\right)-\dot{\Theta} \dot{\psi} \sin \psi, \\
\frac{d Y}{d t}=\dot{Y}, & \frac{d \dot{Y}}{d t}=\sin \psi\left(A_{1} R \cos \psi+A_{2} R \sin \psi+A_{3}\right)+\dot{\Theta} \dot{\psi} \cos \psi, \\
\frac{d \Theta}{d t}=\dot{\Theta}, & \frac{d \dot{\Theta}}{d t}=\left(A_{1} R \cos \psi+A_{2} R \sin \psi+A_{3}\right), \\
\frac{d \psi}{d t}=\dot{\psi}, & \frac{d \dot{\psi}}{d t}=\frac{A_{4}}{B} .
\end{array}
$$

Note that in these new variables, by considering also

$$
Z^{1} \doteq\left(m R^{2}+A\right) z^{1}
$$

the differential system (9.5) obtained by the first method reads

$$
\begin{aligned}
\frac{d X}{d t} & =\cos \psi Z^{1}, & & \\
\frac{d Y}{d t} & =\sin \psi Z^{1}, & \frac{d Z^{1}}{d t} & =A_{1} R \cos \psi+A_{2} R \sin \psi+A_{3} \\
\frac{d \Theta}{d t} & =Z^{1}, & \frac{d z^{2}}{d t} & =B^{-1} A_{4} . \\
\frac{d \psi}{d t} & =z^{2}, & &
\end{aligned}
$$


The two systems (9.7) and (9.8) are in perfect agreement.

The above detailed calculations show that for the rolling disc the first method is much shorter than the second one.

\subsection{Two co-axial rolling discs}

Two identical material discs of radius $R$ running on a plain are joined by a massless common axis, along with they can slide without friction. The configuration manifold is $Q_{6}=\mathbb{R}^{2} \times \mathbb{S}_{1} \times$ $\mathbb{S}_{1} \times \mathbb{S}_{1} \times \mathbb{R}$, with Lagrangian coordinates $\left(q^{1}, q^{2}, q^{3}, q^{4}, q^{5}, q^{6}\right)=\left(x, y, \theta_{1}, \theta_{2}, \psi, a\right)$, where $(x, y)$ are Cartesian coordinates of the center $P_{1}$ of one of the two discs, $\theta_{1}$ and $\theta_{2}$ are the angles of rotations around the common axis, $\psi$ is the angle giving the orientation of the axis, and $a$ is the distance between the centers (see Fig. 4).

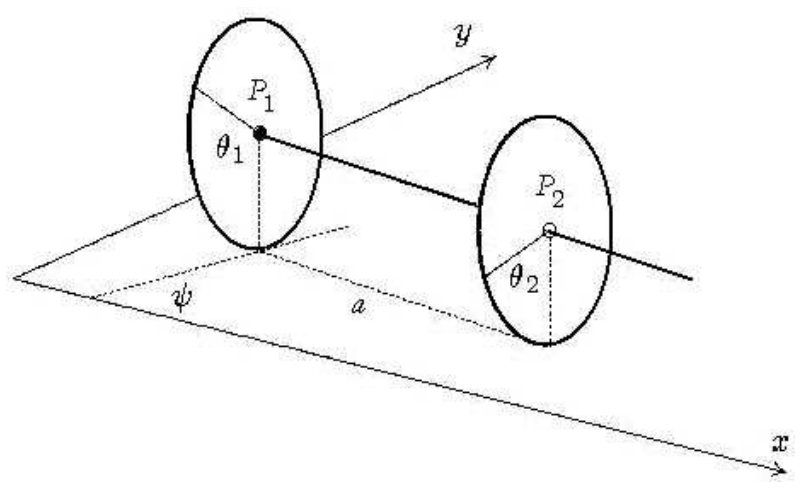

Figure 4. Co-axial rolling discs.

Constraint: the discs roll on the plane without sliding. For each disc this constraint is represented by linear equations of the kind (9.2),

$$
\begin{array}{ll}
\dot{x}_{1}-R \cos \psi \dot{\theta}_{1}=0, & \dot{x}_{2}-R \cos \psi \dot{\theta}_{2}=0, \\
\dot{y}_{1}-R \sin \psi \dot{\theta}_{1}=0, & \dot{y}_{2}-R \sin \psi \dot{\theta}_{2}=0 .
\end{array}
$$

However, the coordinates of the two centers are related by equations

$$
x_{2}=x_{1}+a \sin \psi, \quad y_{2}=y_{1}-a \cos \psi .
$$

By differentiating these equations we get the link between the velocities,

$$
\dot{x}_{2}=\dot{x}_{1}+a \cos \psi \dot{\psi}+\sin \psi \dot{a}, \quad \dot{y}_{2}=\dot{y}_{1}+a \sin \psi \dot{\psi}-\cos \psi \dot{a}
$$

By inserting these relations into equations (9.9), with $x_{1}=x$ and $y_{1}=y$, we get the final constraint equations

$$
\begin{aligned}
& \dot{x}-R \cos \psi \dot{\theta}_{1}=0, \\
& \dot{y}-R \sin \psi \dot{\theta}_{1}=0, \\
& \dot{x}+a \cos \psi \dot{\psi}+\sin \psi \dot{a}-R \cos \psi \dot{\theta}_{2}=0, \\
& \dot{y}+a \sin \psi \dot{\psi}-\cos \psi \dot{a}-R \sin \psi \dot{\theta}_{2}=0
\end{aligned}
$$

Since for a single rolling disc the first method is faster, we limit ourselves to apply the first method to the case of two discs. 
Equations (9.11) show that the constraint submanifold $C \subset T Q_{6}$ has dimension $12-4=8$. Hence, we need two parameters $z^{\alpha}$ for the parametric representation. Let us choose $z^{1}=\dot{\theta}_{1}$ and $z^{2}=\dot{\theta}_{2}$. This means to solve the linear system (9.11) w.r.to $(\dot{x}, \dot{y}, \dot{\psi}, \dot{a})$. The result is

$$
\begin{aligned}
& \dot{x}=R \cos \psi z^{1}, \quad \dot{a}=0, \\
& \dot{y}=R \sin \psi z^{1}, \quad \dot{\theta}_{1}=z^{1} \text {, } \\
& \dot{\psi}=\frac{R}{a}\left(z^{2}-z^{1}\right), \quad \dot{\theta}_{2}=z^{2} .
\end{aligned}
$$

Equation $\dot{a}=0, a=$ constant, exhibits the intuitive fact that, under the pure-rolling condition, the distance $a$ between the two discs remains constant. Hence, we can reduce the configuration manifold $Q_{6}$ to $Q_{5}=\mathbb{R}^{2} \times \mathbb{S}_{1} \times \mathbb{S}_{1} \times \mathbb{S}_{1}$, with coordinates $\left(q^{1}, q^{2}, q^{3}, q^{4}, q^{5}\right)=$ $\left(x, y, \theta_{1}, \theta_{2}, \psi\right)$. The last equations reduce to

$$
\begin{array}{lll}
\dot{x} & =R \cos \psi z^{1}, & \\
\dot{y}=R \sin \psi z^{1}, & \dot{\theta}_{1}=z^{1}, \\
\dot{\psi}=\frac{R}{a}\left(z^{2}-z^{1}\right), & & \dot{\theta}_{2}=z^{2},
\end{array}
$$

with $a=$ constant. Then,

$$
\left[\psi^{i}\right]=\left[R \cos \psi z^{1}, R \sin \psi z^{1}, \frac{R}{a}\left(z^{2}-z^{1}\right), z^{1}, z^{2}\right]
$$

and

$$
\left[\psi_{\alpha}^{i}\right] \doteq\left[\frac{\partial \psi}{\partial z^{\alpha}}\right]=\left[\begin{array}{ccccc}
R \cos \psi & R \sin \psi & -\frac{R}{a} & 1 & 0 \\
0 & 0 & \frac{R}{a} & 0 & 1
\end{array}\right] .
$$

This matrix has maximal rank, thus the constraint submanifold $C$ is regular. The kinetic energy of the system is the sum of the kinetic energies of the two discs. According to equations (9.3) and (9.10),

$$
\begin{aligned}
K= & \frac{1}{2} m\left(\dot{x}_{1}^{2}+\dot{y}_{1}^{2}\right)+\frac{1}{2}\left(A \dot{\theta}_{1}^{2}+B \dot{\psi}^{2}\right)+\frac{1}{2} m\left(\dot{x}_{2}^{2}+\dot{y}_{2}^{2}\right)+\frac{1}{2}\left(A \dot{\theta}_{2}^{2}+B \dot{\psi}^{2}\right) \\
= & \frac{1}{2} m\left(\dot{x}_{1}^{2}+\dot{y}_{1}^{2}+\dot{x}_{2}^{2}+\dot{y}_{2}^{2}\right)+\frac{1}{2}\left(A \dot{\theta}_{1}^{2}+B \dot{\psi}^{2}\right)+\frac{1}{2}\left(A \dot{\theta}_{2}^{2}+B \dot{\psi}^{2}\right) \\
= & \frac{1}{2} m\left(\dot{x}_{1}^{2}+\dot{y}_{1}^{2}+\left(\dot{x}_{1}+a \cos \psi \dot{\psi}\right)^{2}+\left(\dot{y}_{1}+a \sin \psi \dot{\psi}\right)^{2}\right)+\frac{A}{2}\left(\dot{\theta}_{1}^{2}+\dot{\theta}_{2}^{2}\right)+B \dot{\psi}^{2} \\
= & \frac{1}{2} m\left(\dot{x}_{1}^{2}+\dot{y}_{1}^{2}+\dot{x}_{1}^{2}+a^{2} \cos ^{2} \psi \dot{\psi}^{2}+2 a \dot{x}_{1} \cos \psi \dot{\psi}+\dot{y}_{1}^{2}+a^{2} \sin ^{2} \psi \dot{\psi}^{2}\right. \\
& \left.+2 a \dot{y}_{1} \sin \psi \dot{\psi}\right)+\frac{A}{2}\left(\dot{\theta}_{1}^{2}+\dot{\theta}_{2}^{2}\right)+B \dot{\psi}^{2} \\
= & \frac{1}{2} m\left(2 \dot{x}_{1}^{2}+2 \dot{y}_{1}^{2}+a^{2} \dot{\psi}^{2}+2 a \dot{\psi}\left(\dot{x}_{1} \cos \psi+\dot{y}_{1} \sin \psi\right)\right)+\frac{A}{2}\left(\dot{\theta}_{1}^{2}+\dot{\theta}_{2}^{2}\right)+B \dot{\psi}^{2} \\
= & m\left(\dot{x}^{2}+\dot{y}^{2}\right)+\frac{A}{2}\left(\dot{\theta}_{1}^{2}+\dot{\theta}_{2}^{2}\right)+\left(\frac{1}{2} m a^{2}+B\right) \dot{\psi}^{2}+m a \dot{\psi}(\dot{x} \cos \psi+\dot{y} \sin \psi) .
\end{aligned}
$$

Thus,

$$
\left[g_{i j}\right]=\left[\begin{array}{ccccc}
2 m & 0 & 0 & 0 & \frac{m a}{2} \cos \psi \\
0 & 2 m & 0 & 0 & \frac{m a}{2} \sin \psi \\
0 & 0 & A & 0 & 0 \\
0 & 0 & 0 & A & 0 \\
\frac{m a}{2} \cos \psi & \frac{m a}{2} \sin \psi & 0 & 0 & m a^{2}+2 B
\end{array}\right] .
$$


Moreover,

$$
\begin{aligned}
G_{\alpha \beta}= & g_{i j} \psi_{\alpha}^{i} \psi_{\beta}^{j}=g_{11} \psi_{\alpha}^{1} \psi_{\beta}^{1}+g_{22} \psi_{\alpha}^{2} \psi_{\beta}^{2}+g_{33} \psi_{\alpha}^{3} \psi_{\beta}^{3}+g_{44} \psi_{\alpha}^{4} \psi_{\beta}^{4}+g_{55} \psi_{\alpha}^{5} \psi_{\beta}^{5} \\
& +2 g_{12} \psi_{\alpha}^{1} \psi_{\beta}^{2}+2 g_{13} \psi_{\alpha}^{1} \psi_{\beta}^{3}+2 g_{14} \psi_{\alpha}^{1} \psi_{\beta}^{4}+2 g_{15} \psi_{\alpha}^{1} \psi_{\beta}^{5}+2 g_{23} \psi_{\alpha}^{2} \psi_{\beta}^{3}+2 g_{24} \psi_{\alpha}^{2} \psi_{\beta}^{4} \\
& +2 g_{25} \psi_{\alpha}^{2} \psi_{\beta}^{5}+2 g_{34} \psi_{\alpha}^{3} \psi_{\beta}^{4}+2 g_{35} \psi_{\alpha}^{3} \psi_{\beta}^{5}+2 g_{45} \psi_{\alpha}^{4} \psi_{\beta}^{5} \\
= & 2 m \psi_{\alpha}^{1} \psi_{\beta}^{1}+2 m \psi_{\alpha}^{2} \psi_{\beta}^{2}+A \psi_{\alpha}^{3} \psi_{\beta}^{3}+A \psi_{\alpha}^{4} \psi_{\beta}^{4}+\left(m a^{2}+2 B\right) \psi_{\alpha}^{5} \psi_{\beta}^{5} \\
& +m a \cos \psi \psi_{\alpha}^{1} \psi_{\beta}^{5}+m a \sin \psi \psi_{\alpha}^{2} \psi_{\beta}^{5}, \\
G_{11}= & 2 m \psi_{1}^{1} \psi_{1}^{1}+2 m \psi_{1}^{2} \psi_{1}^{2}+A \psi_{1}^{3} \psi_{1}^{3}+A \psi_{1}^{4} \psi_{1}^{4}+\left(m a^{2}+2 B\right) \psi_{1}^{5} \psi_{1}^{5} \\
& +m a \cos \psi \psi_{1}^{1} \psi_{1}^{5}+m a \sin \psi \psi_{1}^{2} \psi_{1}^{5} \\
= & 2 m R^{2} \cos { }^{2} \psi+2 m R^{2} \sin ^{2} \psi+A \frac{R^{2}}{a^{2}}+A=2 m R^{2}+A\left(1+\frac{R^{2}}{a^{2}}\right), \\
G_{22}= & 2 m \psi_{2}^{1} \psi_{2}^{1}+2 m \psi_{2}^{2} \psi_{2}^{2}+A \psi_{2}^{3} \psi_{2}^{3}+A \psi_{2}^{4} \psi_{2}^{4}+\left(m a^{2}+2 B\right) \psi_{2}^{5} \psi_{2}^{5} \\
& +m a \cos \psi \psi_{2}^{1} \psi_{2}^{5}+m a \sin \psi \psi_{2}^{2} \psi_{2}^{5}=A \frac{R^{2}}{a^{2}}+m a^{2}+2 B, \\
G_{12}= & 2 m \psi_{1}^{1} \psi_{2}^{1}+2 m \psi_{1}^{2} \psi_{2}^{2}+A \psi_{1}^{3} \psi_{2}^{3}+A \psi_{1}^{4} \psi_{2}^{4}+\left(m a^{2}+2 B\right) \psi_{1}^{5} \psi_{2}^{5} \\
& +m a \cos \psi \psi_{1}^{1} \psi_{2}^{5}+m a \sin \psi \psi_{1}^{2} \psi_{2}^{5} \\
= & -A \frac{R^{2}}{a^{2}}+m a \cos \psi R \cos \psi+m a \sin \psi R \sin \psi=m a R-A \frac{R^{2}}{a^{2}},
\end{aligned}
$$

and we obtain

$$
\left[G_{\alpha \beta}\right]=\left[\begin{array}{cc}
2 m R^{2}+A\left(1+\frac{R^{2}}{a^{2}}\right) & m a R-A \frac{R^{2}}{a^{2}} \\
m a R-A \frac{R^{2}}{a^{2}} & A \frac{R^{2}}{a^{2}}+m a^{2}+2 B
\end{array}\right] .
$$

It follows that

$$
\begin{aligned}
G \doteq & \operatorname{det}\left[G_{\alpha \beta}\right]=\left[2 m R^{2}+A\left(1+\frac{R^{2}}{a^{2}}\right)\right]\left[A \frac{R^{2}}{a^{2}}+m a^{2}+2 B\right]-\left[m a R-A \frac{R^{2}}{a^{2}}\right]^{2} \\
= & 2 m A \frac{R^{4}}{a^{2}}+2 m^{2} R^{2} a^{2}+4 m R^{2} B+A^{2} \frac{R^{2}}{a^{2}}+m a^{2} A+2 A B+A^{2} \frac{R^{4}}{a^{4}}+m a^{2} A \frac{R^{2}}{a^{2}} \\
& +2 A B \frac{R^{2}}{a^{2}}-m^{2} a^{2} R^{2}-A^{2} \frac{R^{4}}{a^{4}}+2 m A \frac{R^{3}}{a} \\
= & \frac{R^{2}}{a^{2}}\left(2 m A R^{2}+A^{2}+m a^{2} A+2 A B+2 m A a R-m^{2} a^{4}+2 m^{2} a^{4}+4 m a^{2} B\right) \\
& +A\left(m a^{2}+2 B\right) \\
= & \frac{R^{2}}{a^{2}}\left(2 m A R^{2}+A^{2}+m a^{2} A+2 A B+2 m A a R+m^{2} a^{4}+4 m a^{2} B\right)+A\left(m a^{2}+2 B\right) \\
= & \frac{R^{2}}{a^{2}}\left(m\left(2 A R^{2}+a^{2} A+2 A a R+4 a^{2} B+m a^{4}\right)+A^{2}+2 A B\right)+A\left(m a^{2}+2 B\right) .
\end{aligned}
$$

We observe that the determinant $G$ is a constant. The inverse matrix is

$$
\left[G^{\alpha \beta}\right]=G^{-1}\left[\begin{array}{cc}
A \frac{R^{2}}{a^{2}}+m a^{2}+2 B & A \frac{R^{2}}{a^{2}}-m a R \\
A \frac{R^{2}}{a^{2}}-m a R & 2 m R^{2}+A\left(1+\frac{R^{2}}{a^{2}}\right)
\end{array}\right] .
$$

From the expression of the kinetic energy,

$$
K=m\left(\dot{x}^{2}+\dot{y}^{2}\right)+\frac{A}{2}\left(\dot{\theta}_{1}^{2}+\dot{\theta}_{2}^{2}\right)+\left(\frac{1}{2} m a^{2}+B\right) \dot{\psi}^{2}+m a \dot{\psi}(\dot{x} \cos \psi+\dot{y} \sin \psi),
$$


we obtain

$$
\left[\frac{\partial K}{\partial \dot{q}^{i}}\right]=\left[\begin{array}{c}
2 m \dot{x}+m a \cos \psi \dot{\psi} \\
2 m \dot{y}+m a \sin \psi \dot{\psi} \\
A \dot{\theta}_{1} \\
A \dot{\theta}_{2} \\
\left(m a^{2}+2 B\right) \dot{\psi}+m a(\dot{x} \cos \psi+\dot{y} \sin \psi)
\end{array}\right]
$$

and

$$
\left[\frac{\partial K}{\partial q^{i}}\right]=\left[\begin{array}{c}
0 \\
0 \\
0 \\
0 \\
\operatorname{ma} \dot{\psi}(\dot{y} \cos \psi-\dot{x} \sin \psi)
\end{array}\right] .
$$

The Lagrange equations for the free motions are

$$
\begin{aligned}
& 2 m \ddot{x}+m a \cos \psi \ddot{\psi}-m a \sin \psi \dot{\psi}^{2}=A_{1}, \\
& 2 m \ddot{y}+m a \sin \psi \ddot{\psi}+m a \cos \psi \dot{\psi}^{2}=A_{2} \text {, } \\
& A \ddot{\theta}_{1}=A_{3} \text {, } \\
& A \ddot{\theta}_{2}=A_{4} \text {, } \\
& \left(m a^{2}+2 B\right) \ddot{\psi}+m a(\ddot{x} \cos \psi+\ddot{y} \sin \psi)-m a(\dot{x} \sin \psi-\dot{y} \cos \psi) \dot{\psi} \\
& =\operatorname{ma} \dot{\psi}(\dot{y} \cos \psi-\dot{x} \sin \psi)+A_{5} \text {. }
\end{aligned}
$$

They show that

$$
\begin{aligned}
& L_{1}=A_{1}+m a \sin \psi \dot{\psi}^{2}, \\
& L_{2}=A_{2}-m a \cos \psi \dot{\psi}^{2}, \\
& L_{3}=A_{3}, \\
& L_{4}=A_{4}, \\
& L_{5}=A_{5}+m a \dot{\psi}(\dot{y} \cos \psi-\dot{x} \sin \psi)+m a(\dot{x} \sin \psi-\dot{y} \cos \psi) \dot{\psi}=A_{5} .
\end{aligned}
$$

Thus, due to the parametric equations (9.12),

$$
\begin{aligned}
& \bar{Z}_{1}=A_{1}+\frac{m R^{2}}{a}\left(z^{2}-z^{1}\right)^{2} \sin \psi, \quad \bar{Z}_{2}=A_{2}-\frac{m R^{2}}{a}\left(z^{2}-z^{1}\right)^{2} \cos \psi, \\
& \bar{Z}_{3}=A_{3}, \quad \bar{Z}_{4}=A_{4}, \quad \bar{Z}_{5}=A_{5} .
\end{aligned}
$$

Let us compute $Z_{\alpha}=\bar{Z}_{i} \psi_{\alpha}^{i}-$ recall (9.13):

$$
\begin{aligned}
Z_{1}= & \left(A_{1}+\frac{m R^{2}}{a}\left(z^{2}-z^{1}\right)^{2} \sin \psi\right) R \cos \psi+\left(A_{2}-\frac{m R^{2}}{a}\left(z^{2}-z^{1}\right)^{2} \cos \psi\right) R \sin \psi \\
& -\frac{R}{a} A_{3}+A_{4} \\
= & A_{1} R \cos \psi+A_{2} R \sin \psi-\frac{R}{a} A_{3}+A_{4}=R\left(A_{1} \cos \psi+A_{2} \sin \psi-\frac{1}{a} A_{3}\right)+A_{4}, \\
Z_{2}= & \frac{R}{a} A_{3}+A_{5} .
\end{aligned}
$$


Thus, the dynamical equations (7.5) associated with the vector field $\mathbf{Z}$ are

$$
\begin{array}{rlrl}
\frac{d x}{d t} & =R \cos \psi z^{1}, & & \\
\frac{d x}{d t} & =R \sin \psi z^{1}, & \frac{d z^{1}}{d t}=R\left(A_{1} \cos \psi+A_{2} \sin \psi-\frac{1}{a} A_{3}\right)+A_{4} \\
\frac{d \psi}{d t}=\frac{R}{a}\left(z^{2}-z^{1}\right), & \frac{d z^{2}}{d t}=\frac{R}{a} A_{3}+A_{5} \\
\frac{d \theta_{1}}{d t}=z^{1}, & \\
\frac{d \theta_{2}}{d t}=z^{2} &
\end{array}
$$

\subsection{Two points with parallel velocities}

Two material points $P_{1}=\left(x_{1}, y_{1}\right)$ and $P_{2}=\left(x_{2}, y_{2}\right)$ running on the Cartesian plane $\mathbb{R}^{2}=(x, y)$ are constrained to have parallel vector-velocities $\mathbf{v}_{1}$ and $\mathbf{v}_{2}$. This is an example of non-linear non-holonomic constraint, since it is expressed by the quadratic homogeneous equation

$$
C=\dot{x}_{1} \dot{y}_{2}-\dot{x}_{2} \dot{y}_{1}=0 \text {. }
$$

The configuration manifold is $Q_{4}=\mathbf{R}^{4}$ with ordered Lagrangian coordinates

$$
\left(q^{1}, q^{2}, q^{3}, q^{4}\right)=\left(x_{1}, y_{1}, x_{2}, y_{2}\right)
$$

The kinetic energy is $K=\frac{1}{2} m_{1}\left(\dot{x}_{1}^{2}+\dot{y}_{1}^{2}\right)+\frac{1}{2} m_{2}\left(\dot{x}_{2}^{2}+\dot{y}_{2}^{2}\right)$. Hence,

$$
\left[g_{i j}\right]=\left[\begin{array}{cccc}
m_{1} & 0 & 0 & 0 \\
0 & m_{1} & 0 & 0 \\
0 & 0 & m_{2} & 0 \\
0 & 0 & 0 & m_{2}
\end{array}\right]
$$

(i) First method. Since $\operatorname{dim}(Q)=4$ and $\operatorname{dim}(C)=7$, for a parametric representation of the constraint $\dot{x}_{1} \dot{y}_{2}-\dot{x}_{2} \dot{y}_{1}=0$ we need three parameters $\left(z^{1}, z^{2}, z^{3}\right)$. Let us consider the parameters $\left(z^{\alpha}\right)=\left(z^{1}, z^{2}, z^{3}\right)=(\rho, \sigma, \theta)$ and the parametric equations

$$
\begin{array}{ll}
\dot{x}_{1}=\rho \cos \theta, & \dot{x}_{2}=\sigma \cos \theta, \\
\dot{y}_{1}=\rho \sin \theta, & \dot{y}_{2}=\sigma \sin \theta .
\end{array}
$$

The meaning of the parameters is the following: $\rho^{2}=\mathbf{v}_{1}^{2}=\dot{x}_{1}^{2}+\dot{y}_{1}^{2}, \sigma^{2}=\mathbf{v}_{2}^{2}=\dot{x}_{2}^{2}+\dot{y}_{2}^{2}$, and $\theta$ is the angle of the two vector velocities w.r.to the $x$-axis. Then we find:

$$
\begin{aligned}
& {\left[\psi_{\alpha}^{i}\right]=\left[\begin{array}{cccc}
\cos \theta & \sin \theta & 0 & 0 \\
0 & 0 & \cos \theta & \sin \theta \\
-\rho \sin \theta & \rho \cos \theta & -\sigma \sin \theta & \sigma \cos \theta
\end{array}\right] \quad \text { ( } \alpha \text { index of line), }} \\
& {\left[G_{\alpha \beta}\right] \doteq\left[g_{i j} \psi_{\alpha}^{i} \psi_{\beta}^{j}\right]=\left[\begin{array}{ccc}
m_{1} & 0 & 0 \\
0 & m_{2} & 0 \\
0 & 0 & m_{1} \rho^{2}+m_{2} \sigma^{2}
\end{array}\right],} \\
& {\left[G^{\alpha \beta}\right]=\left[\begin{array}{ccc}
\frac{1}{m_{1}} & 0 & 0 \\
0 & \frac{1}{m_{2}} & 0 \\
0 & 0 & \frac{1}{m_{1} \rho^{2}+m_{2} \sigma^{2}}
\end{array}\right]}
\end{aligned}
$$


Since,

$$
\left[\frac{\partial K}{\partial \dot{q}^{i}}\right]=\left[m_{1} \dot{x}_{1}, m_{1} \dot{y}_{1}, m_{2} \dot{x}_{2}, m_{2} \dot{y}_{2}\right], \quad\left[\frac{\partial K}{\partial q^{i}}\right]=[0,0,0,0],
$$

the Lagrange equations for the free motions $g_{i j} \ddot{q}^{j}=A_{i}-\Gamma_{h k i} \dot{q}^{h} \dot{q}^{k}$ read

$$
m_{1} \ddot{x}_{1}=A_{1}, \quad m_{1} \ddot{y}_{1}=A_{2}, \quad m_{2} \ddot{x}_{2}=A_{3}, \quad m_{2} \ddot{y}_{2}=A_{4} .
$$

They show that $\bar{Z}_{i}=A_{i}$. Hence,

$$
\begin{gathered}
{\left[Z_{\alpha}\right] \doteq\left[\psi_{\alpha}^{i} \bar{Z}_{i}\right]=\left[\begin{array}{c}
A_{1} \cos \theta+A_{2} \sin \theta \\
A_{3} \cos \theta+A_{4} \sin \theta \\
\rho\left(A_{2} \cos \theta-A_{1} \sin \theta\right)+\sigma\left(A_{4} \cos \theta-A_{3} \sin \theta\right)
\end{array}\right],} \\
{\left[Z^{\alpha}\right] \doteq\left[G^{\alpha \beta} Z_{\beta}\right]=\left[\begin{array}{c}
\frac{A_{1} \cos \theta+A_{2} \sin \theta}{m_{1}} \\
\frac{A_{3} \cos \theta+A_{4} \sin \theta}{m_{2}} \\
\frac{\rho\left(A_{2} \cos \theta-A_{1} \sin \theta\right)+\sigma\left(A_{4} \cos \theta-A_{3} \sin \theta\right)}{m_{1} \rho^{2}+m_{2} \sigma^{2}}
\end{array}\right],}
\end{gathered}
$$

and the differential system associated with $\mathbf{Z}$ is

$$
\begin{array}{rlrl}
\frac{d x_{1}}{d t} & =\rho \cos \theta, & & \frac{d \rho}{d t}=\frac{A_{1} \cos \theta+A_{2} \sin \theta}{m_{1}}, \\
\frac{d y_{1}}{d t}=\rho \sin \theta, & \frac{d \sigma}{d t}=\frac{A_{3} \cos \theta+A_{4} \sin \theta}{m_{2}}, \\
\frac{d x_{2}}{d t}=\sigma \cos \theta, & \frac{d \theta}{d t}=\frac{\rho\left(A_{2} \cos \theta-A_{1} \sin \theta\right)+\sigma\left(A_{4} \cos \theta-A_{3} \sin \theta\right)}{m_{1} \rho^{2}+m_{2} \sigma^{2}}, \\
\frac{d y_{2}}{d t}=\sigma \sin \theta, &
\end{array}
$$

where the Lagrangian active forces are in general known functions of $\left(x_{1}, y_{1}, x_{2}, y_{2}\right)$ and $(\rho, \sigma, \theta)$.

In the special case of an inclined plane we have $A_{1}=m_{1} g, A_{3}=m_{2} g, A_{2}=A_{4}=0$, and the system (9.17) becomes

$$
\begin{array}{rlrl}
\frac{d x_{1}}{d t} & =\rho \cos \theta, & & \frac{d \rho}{d t}=g \cos \theta, \\
\frac{d y_{1}}{d t}=\rho \sin \theta, & \frac{d \sigma}{d t}=g \cos \theta, \\
\frac{d x_{2}}{d t}=\sigma \cos \theta, & \frac{d \theta}{d t}=-g \sin \theta \frac{m_{1} \rho+m_{2} \sigma}{m_{1} \rho^{2}+m_{2} \sigma^{2}} . \\
\frac{d y_{2}}{d t}=\sigma \sin \theta, & &
\end{array}
$$

Note that the last three equations are separated from the first four. This occurs in general when the Lagrangian active forces do not depend on the position of the point, but only on their velocities. For equal masses $m_{1}=m_{2}$, we have a further simplification:

$$
\begin{array}{rlrl}
\frac{d x_{1}}{d t} & =\rho \cos \theta, & & \frac{d \rho}{d t}=g \cos \theta, \\
\frac{d y_{1}}{d t}=\rho \sin \theta, & \frac{d \sigma}{d t}=g \cos \theta, \\
\frac{d x_{2}}{d t}=\sigma \cos \theta, & \frac{d \theta}{d t}=-g \sin \theta \frac{\rho+\sigma}{\rho^{2}+\sigma^{2}} . \\
\frac{d y_{2}}{d t}=\sigma \sin \theta, &
\end{array}
$$


(ii) Second method for the single constraint equation (9.14). In this case,

$$
\left[C_{i}\right]=\left[\dot{y}_{2},-\dot{x}_{2},-\dot{y}_{1}, \dot{x}_{1}\right]
$$

does not have the maximal rank for $\mathbf{v}_{1}=\mathbf{v}_{2}=0$. This is a singular state for whatever configuration (see Remark 7.3); the set of the singular states is the zero-section of $T Q$. Moreover, since

$$
\left[g^{i j}\right]=\left[\begin{array}{cccc}
\frac{1}{m_{1}} & 0 & 0 & 0 \\
0 & \frac{1}{m_{1}} & 0 & 0 \\
0 & 0 & \frac{1}{m_{2}} & 0 \\
0 & 0 & 0 & \frac{1}{m_{2}}
\end{array}\right]
$$

we have:

$$
\begin{aligned}
& {\left[C^{i}\right] \doteq\left[g^{i j} C_{j}\right]=\left[\frac{\dot{y}_{2}}{m_{1}},-\frac{\dot{x}_{2}}{m_{1}},-\frac{\dot{y}_{1}}{m_{2}}, \frac{\dot{x}_{1}}{m_{2}}\right],} \\
& G=\frac{\dot{x}_{2}^{2}+\dot{y}_{2}^{2}}{m_{1}}+\frac{\dot{x}_{1}^{2}+\dot{y}_{1}^{2}}{m_{2}}=\frac{2 K}{m_{1} m_{2}}, \quad G^{-1}=\frac{m_{1} m_{2}}{2 K}, \\
& {\left[C_{*}^{i}\right]=G^{-1}\left[\frac{\dot{y}_{2}}{m_{1}},-\frac{\dot{x}_{2}}{m_{1}},-\frac{\dot{y}_{1}}{m_{2}}, \frac{\dot{x}_{1}}{m_{2}}\right],} \\
& {\left[\pi^{i j}\right]=G^{-1}\left[\begin{array}{cccc}
\frac{\dot{y}_{2}^{2}}{m_{1}^{2}} & -\frac{\dot{x}_{2} \dot{y}_{2}}{m_{1}^{2}} & -\frac{\dot{y}_{1} \dot{y}_{2}}{m_{1} m_{2}} & \frac{\dot{x}_{1} \dot{y}_{2}}{m_{1} m_{2}} \\
-\frac{\dot{x}_{2} \dot{y}_{2}}{m_{1}^{2}} & \frac{\dot{x}_{2}^{2}}{m_{1}^{2}} & \frac{\dot{x}_{2} \dot{y}_{1}}{m_{1} m_{2}} & -\frac{\dot{x}_{1} \dot{x}_{2}}{m_{1} m_{2}} \\
-\frac{\dot{y}_{1} \dot{y}_{2}}{m_{1} m_{2}} & \frac{\dot{y}_{1} \dot{x}_{2}}{m_{1} m_{2}} & \frac{\dot{y}_{1}^{2}}{m_{2}^{2}} & -\frac{\dot{x}_{1} \dot{y}_{1}}{m_{2}^{2}} \\
\frac{\dot{x}_{1} \dot{y}_{2}}{m_{1} m_{2}} & -\frac{\dot{x}_{1} \dot{x}_{2}}{m_{1} m_{2}} & -\frac{\dot{x}_{1} \dot{y}_{1}}{m_{2}^{2}} & \frac{\dot{x}_{1}^{2}}{m_{2}^{2}}
\end{array}\right],} \\
& {\left[g^{i j}-\pi^{i j}\right]=\left[\begin{array}{cccc}
\frac{1}{m_{1}} & 0 & 0 & 0 \\
0 & \frac{1}{m_{1}} & 0 & 0 \\
0 & 0 & \frac{1}{m_{2}} & 0 \\
0 & 0 & 0 & \frac{1}{m_{2}}
\end{array}\right]} \\
& -\frac{m_{1} m_{2}}{2 K}\left[\begin{array}{cccc}
\frac{\dot{y}_{2}^{2}}{m_{1}^{2}} & -\frac{\dot{x}_{2} \dot{y}_{2}}{m_{1}^{2}} & -\frac{\dot{y}_{1} \dot{y}_{2}}{m_{1} m_{2}} & \frac{\dot{x}_{1} \dot{y}_{2}}{m_{1} m_{2}} \\
-\frac{\dot{x}_{2} \dot{y}_{2}}{m_{1}^{2}} & \frac{\dot{x}_{2}^{2}}{m_{1}^{2}} & \frac{\dot{x}_{2} \dot{y}_{1}}{m_{1} m_{2}} & -\frac{\dot{x}_{1} \dot{x}_{2}}{m_{1} m_{2}} \\
-\frac{\dot{y}_{1} \dot{y}_{2}}{m_{1} m_{2}} & \frac{\dot{y}_{1} \dot{x}_{2}}{m_{1} m_{2}} & \frac{\dot{y}_{1}^{2}}{m_{2}^{2}} & -\frac{\dot{x}_{1} \dot{y}_{1}}{m_{2}^{2}} \\
\frac{\dot{x}_{1} \dot{y}_{2}}{m_{1} m_{2}} & -\frac{\dot{x}_{1} \dot{x}_{2}}{m_{1} m_{2}} & -\frac{\dot{x}_{1} \dot{y}_{1}}{m_{2}^{2}} & \frac{\dot{x}_{1}^{2}}{m_{2}^{2}}
\end{array}\right], \\
& g^{11}-\pi^{11}=\frac{1}{m_{1}}-\frac{m_{2}}{2 K} \frac{\dot{y}_{2}^{2}}{m_{1}}=\frac{1}{m_{1}}\left(1-\frac{m_{2} \dot{y}_{2}^{2}}{2 K}\right)=\frac{2 K-m_{2} \dot{y}_{2}^{2}}{2 m_{1} K}=\frac{m_{1}\left(\dot{x}_{1}^{2}+\dot{y}_{1}^{2}\right)+m_{2} \dot{x}_{2}^{2}}{2 m_{1} K} \text {, } \\
& g^{12}-\pi^{12}=\frac{m_{1} m_{2}}{2 K} \frac{\dot{x}_{2} \dot{y}_{2}}{m_{1}^{2}}=\frac{m_{2} \dot{x}_{2} \dot{y}_{2}}{2 m_{1} K},
\end{aligned}
$$




$$
\begin{aligned}
& g^{13}-\pi^{13}=\frac{m_{1} m_{2}}{2 K} \frac{\dot{y}_{1} \dot{y}_{2}}{m_{1} m_{2}}=\frac{\dot{y}_{1} \dot{y}_{2}}{2 K}, \\
& g^{14}-\pi^{14}=-\frac{m_{1} m_{2}}{2 K} \frac{\dot{x}_{1} \dot{y}_{2}}{m_{1} m_{2}}=-\frac{\dot{x}_{1} \dot{y}_{2}}{2 K}, \\
& g^{22}-\pi^{22}=\frac{1}{m_{1}}-\frac{m_{2}}{2 K} \frac{\dot{x}_{2}^{2}}{m_{1}}=\frac{1}{m_{1}}\left(1-\frac{m_{2} \dot{x}_{2}^{2}}{2 K}\right)=\frac{2 K-m_{2} \dot{x}_{2}^{2}}{2 m_{1} K}=\frac{m_{1}\left(\dot{x}_{1}^{2}+\dot{y}_{1}^{2}\right)+m_{2} \dot{y}_{2}^{2}}{2 m_{1} K}, \\
& g^{23}-\pi^{23}=-\frac{m_{1} m_{2}}{2 K} \frac{\dot{x}_{2} \dot{y}_{1}}{m_{1} m_{2}}=-\frac{\dot{x}_{2} \dot{y}_{1}}{2 K}, \\
& g^{24}-\pi^{24}=\frac{m_{1} m_{2}}{2 K} \frac{\dot{x}_{1} \dot{x}_{2}}{m_{1} m_{2}}=\frac{\dot{x}_{1} \dot{x}_{2}}{2 K}, \\
& g^{33}-\pi^{33}=\frac{1}{m_{2}}-\frac{m_{1}}{2 K} \frac{\dot{y}_{1}^{2}}{m_{2}}=\frac{1}{m_{2}}\left(1-\frac{m_{1} \dot{y}_{1}^{2}}{2 K}\right)=\frac{2 K-m_{1} \dot{y}_{1}^{2}}{2 m_{2} K}=\frac{m_{2}\left(\dot{x}_{2}^{2}+\dot{y}_{2}^{2}\right)+m_{1} \dot{x}_{1}^{2}}{2 m_{2} K}, \\
& g^{34}-\pi^{34}=\frac{m_{1} m_{2}}{2 K} \frac{\dot{x}_{1} \dot{y}_{1}}{m_{2}^{2}}=\frac{m_{1} \dot{x}_{1} \dot{y}_{1}}{2 m_{2} K}, \\
& g^{44}-\pi^{44}=\frac{1}{m_{2}}-\frac{m_{1}}{2 K} \frac{\dot{x}_{1}^{2}}{m_{2}}=\frac{1}{m_{2}}\left(1-\frac{m_{1} \dot{x}_{1}^{2}}{2 K}\right)=\frac{2 K-m_{1} \dot{x}_{1}^{2}}{2 m_{2} K}=\frac{m_{2}\left(\dot{x}_{2}^{2}+\dot{y}_{2}^{2}\right)+m_{1} \dot{y}_{1}^{2}}{2 m_{2} K}, \\
& {\left[\partial_{i} C\right]=[0,0,0,0], \quad\left[\partial_{j} C C_{*}^{i}\right]=[\mathbf{0}] .}
\end{aligned}
$$

As in this case $L_{i}=A_{i}$, we have

$$
\begin{aligned}
D^{i} & \doteq\left(g^{i j}-\pi^{i j}\right) L_{j}-\dot{q}^{j} \partial_{j} C C_{*}^{i}=\left(g^{i j}-\pi^{i j}\right) L_{j}=\left(g^{i j}-\pi^{i j}\right) A_{j}, \\
D^{1} & =\frac{1}{2 K}\left(A_{1} \frac{m_{1}\left(\dot{x}_{1}^{2}+\dot{y}_{1}^{2}\right)+m_{2} \dot{x}_{2}^{2}}{m_{1}}+A_{2} \frac{m_{2} \dot{x}_{2} \dot{y}_{2}}{m_{1}}+A_{3} \dot{y}_{1} \dot{y}_{2}-A_{4} \dot{x}_{1} \dot{y}_{2}\right), \\
D^{2} & =\frac{1}{2 K}\left(A_{1} \frac{m_{2} \dot{x}_{2} \dot{y}_{2}}{m_{1}}+A_{2} \frac{m_{1}\left(\dot{x}_{1}^{2}+\dot{y}_{1}^{2}\right)+m_{2} \dot{y}_{2}^{2}}{m_{1}}-A_{3} \dot{x}_{2} \dot{y}_{1}+A_{4} \dot{x}_{1} \dot{x}_{2}\right), \\
D^{3} & =\frac{1}{2 K}\left(A_{1} \dot{y}_{1} \dot{y}_{2}-A_{2} \dot{x}_{2} \dot{y}_{1}+A_{3} \frac{m_{2}\left(\dot{x}_{2}^{2}+\dot{y}_{2}^{2}\right)+m_{1} \dot{x}_{1}^{2}}{m_{2}}+A_{4} \frac{m_{1} \dot{x}_{1} \dot{y}_{1}}{m_{2}}\right), \\
D^{4} & =\frac{1}{2 K}\left(-A_{1} \dot{x}_{1} \dot{y}_{2}+A_{2} \dot{x}_{1} \dot{x}_{2}+A_{3} \frac{m_{1} \dot{x}_{1} \dot{y}_{1}}{m_{2}}+A_{4} \frac{m_{2}\left(\dot{x}_{2}^{2}+\dot{y}_{2}^{2}\right)+m_{1} \dot{y}_{1}^{2}}{m_{2}}\right) .
\end{aligned}
$$

Then the dynamical system (8.12) reads

$$
\begin{aligned}
\frac{d x_{1}}{d t} & =\dot{x}_{1}, & \frac{d \dot{x}_{1}}{d t} & =\frac{1}{2 K}\left(A_{1} \frac{m_{1}\left(\dot{x}_{1}^{2}+\dot{y}_{1}^{2}\right)+m_{2} \dot{x}_{2}^{2}}{m_{1}}+A_{2} \frac{m_{2} \dot{x}_{2} \dot{y}_{2}}{m_{1}}+A_{3} \dot{y}_{1} \dot{y}_{2}-A_{4} \dot{x}_{1} \dot{y}_{2}\right), \\
\frac{d y_{1}}{d t} & =\dot{y}_{1}, & \frac{d \dot{y}_{1}}{d t} & =\frac{1}{2 K}\left(A_{1} \frac{m_{2} \dot{x}_{2} \dot{y}_{2}}{m_{1}}+A_{2} \frac{m_{1}\left(\dot{x}_{1}^{2}+\dot{y}_{1}^{2}\right)+m_{2} \dot{y}_{2}^{2}}{m_{1}}-A_{3} \dot{x}_{2} \dot{y}_{1}+A_{4} \dot{x}_{1} \dot{x}_{2}\right), \\
\frac{d x_{2}}{d t} & =\dot{x}_{2}, & \frac{d \dot{x}_{2}}{d t} & =\frac{1}{2 K}\left(A_{1} \dot{y}_{1} \dot{y}_{2}-A_{2} \dot{x}_{2} \dot{y}_{1}+A_{3} \frac{m_{2}\left(\dot{x}_{2}^{2}+\dot{y}_{2}^{2}\right)+m_{1} \dot{x}_{1}^{2}}{m_{2}}+A_{4} \frac{m_{1} \dot{x}_{1} \dot{y}_{1}}{m_{2}}\right), \\
\frac{d y_{2}}{d t} & =\dot{y}_{2}, & \frac{d \dot{y}_{2}}{d t} & =\frac{1}{2 K}\left(-A_{1} \dot{x}_{1} \dot{y}_{2}+A_{2} \dot{x}_{1} \dot{x}_{2}+A_{3} \frac{m_{1} \dot{x}_{1} \dot{y}_{1}}{m_{2}}+A_{4} \frac{m_{2}\left(\dot{x}_{2}^{2}+\dot{y}_{2}^{2}\right)+m_{1} \dot{y}_{1}^{2}}{m_{2}}\right) .
\end{aligned}
$$

For two points running on an inclined plane,

$$
\begin{array}{ll}
D^{1}=g \frac{m_{1}\left(\dot{x}_{1}^{2}+\dot{y}_{1}^{2}\right)+m_{2}\left(\dot{x}_{2}^{2}+\dot{y}_{1} \dot{y}_{2}\right)}{m_{1}\left(\dot{x}_{1}^{2}+\dot{y}_{1}^{2}\right)+m_{2}\left(\dot{x}_{2}^{2}+\dot{y}_{2}^{2}\right)}, & D^{3}=g \frac{m_{2}\left(\dot{x}_{2}^{2}+\dot{y}_{2}^{2}\right)+m_{1}\left(\dot{x}_{1}^{2}+\dot{y}_{1} \dot{y}_{2}\right)}{m_{1}\left(\dot{x}_{1}^{2}+\dot{y}_{1}^{2}\right)+m_{2}\left(\dot{x}_{2}^{2}+\dot{y}_{2}^{2}\right)}, \\
D^{2}=g \frac{m_{2} \dot{x}_{2}\left(\dot{y}_{2}-\dot{y}_{1}\right)}{m_{1}\left(\dot{x}_{1}^{2}+\dot{y}_{1}^{2}\right)+m_{2}\left(\dot{x}_{2}^{2}+\dot{y}_{2}^{2}\right)}, & D^{4}=g \frac{m_{1} \dot{x}_{1}\left(\dot{y}_{1}-\dot{y}_{2}\right)}{m_{1}\left(\dot{x}_{1}^{2}+\dot{y}_{1}^{2}\right)+m_{2}\left(\dot{x}_{2}^{2}+\dot{y}_{2}^{2}\right)} .
\end{array}
$$


For equal masses, $m_{1}=m_{2}$,

$$
\begin{aligned}
D^{1} & =g \frac{\dot{x}_{1}^{2}+\dot{y}_{1}^{2}+\dot{x}_{2}^{2}+\dot{y}_{1} \dot{y}_{2}}{\dot{x}_{1}^{2}+\dot{y}_{1}^{2}+\dot{x}_{2}^{2}+\dot{y}_{2}^{2}}, & D^{3} & =g \frac{\dot{x}_{2}^{2}+\dot{y}_{2}^{2}+\dot{x}_{1}^{2}+\dot{y}_{1} \dot{y}_{2}}{\dot{x}_{1}^{2}+\dot{y}_{1}^{2}+\dot{x}_{2}^{2}+\dot{y}_{2}^{2}}, \\
D^{2} & =g \frac{\dot{x}_{2}\left(\dot{y}_{2}-\dot{y}_{1}\right)}{\dot{x}_{1}^{2}+\dot{y}_{1}^{2}+\dot{x}_{2}^{2}+\dot{y}_{2}^{2}}, & D^{4} & =g \frac{\dot{x}_{1}\left(\dot{y}_{1}-\dot{y}_{2}\right)}{\dot{x}_{1}^{2}+\dot{y}_{1}^{2}+\dot{x}_{2}^{2}+\dot{y}_{2}^{2}} .
\end{aligned}
$$

Note 9.1. It is easy (and obvious) to propose examples of non-linear constraints: it is sufficient to choose any set of non-linear independent equations $C^{a}(q, \dot{q})=0$. However, any example of a non-linear constraint remains meaningless unless we know how to realize it physically by means of realizable devices. The famous Appell-Hamel example gives a matter of discussion (see [9], Ch. 4, § 2). Indeed, in order to be really a non-linear device, a certain distance of the Appell-Hamel device must be infinitesimally small. This fits with the thought of Hertz: nonlinear constraints can be realized by passing to the limit $x \rightarrow 0$ of certain physical quantities $x$ (masses, lengths, etc.) in devices realizing linear constraints.

The same kind of problem arises in trying to 'realize' two mass-points moving with parallel velocities. A tentative project has been presented in [1]. In fact, for an effective project, we have to invent devices for

1. Realizing a mass-point.

2. Realizing a parallel transport on the plane.

3. Transforming the direction of the velocity of a point into a solid segment.

4. Applying forces of special kind to the points (the weight is of course always present).

This research is a work in progress. Updated information will be found on my personal web-page.

\section{Acknowledgments}

A preliminary version of this paper has been elaborated and exposed at the University of Linköping, Department of Mathematics, on May 27, 2005. I wish to thank Stefan Rauch and all the Linköping school for their warm hospitality. I wish also to thank: Waldyr Oliva, Willy Sarlet, David Martin de Diego for making me aware of their contributions to the theory; Enrico Pagani and Enrico Massa, for the enlightening discussions during a workshop on dynamical systems held in Torino in April 2005 - their papers [8, 7] have been of great help; Beppe Gaeta, for pointing me out some errors in my first manuscript; Franco Cardin for his kind invitation to give a seminar in Padova on the contents of this paper (November 2006).

\section{References}

[1] Benenti S., Geometrical aspects of the dynamics of non-holonomic systems, Rend. Sem. Mat. Univ. Pol. Torino 54 (1996), 203-212.

[2] Bullo F., Lewis A.D., Geometric control of mechanical systems, Texts in Applied Mathematics, Vol. 49, Springer, Berlin, 2004.

[3] Carathéodory C., Sur les équations de la mécanique, Actes Congrès Interbalcanian Math. (1934, Athènes), $1935,211-214$.

[4] Cortés Monforte J., Geometrical, control and numerical aspects of nonholonomic systems, Lecture Notes in Mathematics, Vol. 1793, Springer, Berlin, 2002.

[5] Gantmacher F., Lectures in analytical mechanics, Mir, Moscow, 1970.

[6] Marle C.-M., Reduction of constrained mechanical systems and stability of relative equilibria, Comm. Math. Phys. 174 (1995), 295-318. 
[7] Massa E., Pagani E., A new look at classical mechanics of constrained systems, Ann. Inst. H. Poincaré Phys. Théor. 66 (1997), 1-36.

[8] Massa E., Pagani E., Classical dynamics of non-holonomic systems: a geometric approach, Ann. Inst. H. Poincaré Phys. Théor. 55 (1991), 511-544.

[9] Neimark J.I., Fufaev N.A., Dynamics of nonholonomic systems, Translations of Mathematical Monographs, Vol. 33, American Mathematical Society, Providence, Rhode Island, 1972.

[10] Oliva W.M., Kobayashi M.H., A note on the conservation of energy and volume in the setting of nonholonomic mechanical systems, Qual. Theory Dyn. Syst. 4 (2004), 383-411. 